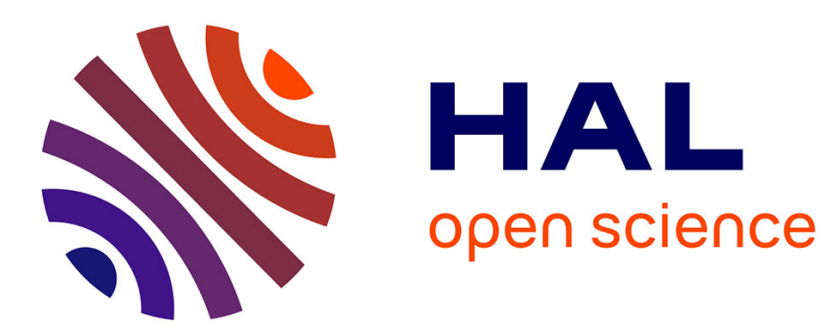

\title{
Dynamic output feedback for switched linear systems based on a LQG design
}

Pierre Riedinger, Jean-Claude Vivalda

\section{To cite this version:}

Pierre Riedinger, Jean-Claude Vivalda. Dynamic output feedback for switched linear systems based on a LQG design. Automatica, 2015, 54, pp.235-245. 10.1016/j.automatica.2015.02.007 . hal-01138922

\section{HAL Id: hal-01138922 \\ https://hal.science/hal-01138922}

Submitted on 3 Apr 2015

HAL is a multi-disciplinary open access archive for the deposit and dissemination of scientific research documents, whether they are published or not. The documents may come from teaching and research institutions in France or abroad, or from public or private research centers.
L'archive ouverte pluridisciplinaire HAL, est destinée au dépôt et à la diffusion de documents scientifiques de niveau recherche, publiés ou non, émanant des établissements d'enseignement et de recherche français ou étrangers, des laboratoires publics ou privés. 


\title{
Dynamic output feedback for switched linear systems based on a LQG design
}

\author{
Pierre Riedinger ${ }^{\mathrm{a}}$ Jean-Claude Vivalda ${ }^{\mathrm{b}}$ \\ ${ }^{a}$ CRAN, Université de Lorraine, CRAN, UMR 7039, 2, avenue de la forêt de Haye, Vandoeuvre-lès-Nancy Cedex, 54516, \\ France - CNRS, CRAN, UMR 7039, France \\ ${ }^{\mathrm{b}}$ Inria, CORIDA, Villers-lès-Nancy, F-54600, France - Université de Lorraine, Institut Elie Cartan de Lorraine, UMR 7502, \\ Vandoeuvre-lès-Nancy, F-54506, France-CNRS, Institut Elie Cartan de Lorraine, UMR 7502, Vandouvre-lès-Nancy, \\ F-54506, France
}

\begin{abstract}
The aim of this paper is the extend of the LQG design for linear system to the case of switched linear systems in continuous time. The main result provides a control Lyapunov function and a dynamic output feedback law leading to sub-optimal solutions. Practically, the dynamic output feedback is easy to apply and the design procedure is effective if there exists at least one controllable and observable convex combination of the subsystems. Practical applications concern the large class of power converters.
\end{abstract}

Key words: Output feedback, LQG, Switched systems, DC-DC Power converters

\section{Introduction}

Over the past decade, the design of optimal control laws for switched systems (in continuous and discrete time) has been the focus of considerable research attention. Several approaches have been used to tackle this problem, one can cite for example $[18,22,24,25]$ for dynamic programming approaches, $[1,3,23]$ for variational approaches, or $[5,9,11]$ for Lyapunov based approaches. This problem is not easy, even numerically [20,23] and the design of a stabilizing feedback law based on the optimization of a criterion is a challenging task.

LQ regulators are widely used for the control of linear systems because of their simple design and their robustness and performance properties. When full state measurement is not available, the LQG design that uses, in addition, an asymptotic Kalman filter to estimate the state, is a valid alternative due to the separation principle that guarantees the global asymptotic stability. For switched systems, there is no separation principle and the stability is highly related of the chosen switching law.

Most of the results in the literature are devoted to the stability analysis of switching systems in discrete time where the stability is established, whatever the switching laws, thanks to the use of LMI conditions [6]. An- other approach consists in investigating the stable and unstable subspace related to each matrix $A_{i}$, in [26], the authors give a sufficient condition condition for the existence of an exponential stabilizing law; this condition bears on the relative positions these stable and unstable subspaces. For cases where this design is not relevant (at least a switching law is unstable) or in order to improve performance, a more involved problem is the design of a stabilizing control law. Less results deal with this latter problem $[11,17]$ and, in particular, very little attention has been devoted to the design of output feedback control law. In $[12,8]$, the stabilizing design procedures are based on the determination of a piecewise quadratic Lyapunov function. More precisely, a set of LyapunovMetzler inequalities provides conditions for state and output feedback. As the method is related to the solvability of LMI / BMI conditions depending on parameter design, it possess intrinsically a conservative part and practically the tractability is not obvious.

In recent papers [21], a switched LQ design has been developed to meet some performance requirements based on the minimization of a switched quadratic cost function. It has been shown that the provided stabilizing feedback law derived from a control Lyapunov function (not necessarily convex), approaches the optimal one in a sense precise in section 2 . The only requirement for applying the method is the existence of one controllable 
convex combination of the subsystems. The appealing side of the method lies also in its practical aspect since only a set of algebraic Riccati equations have to be solved to establish the Lyapunov function.

In this paper, our aim is to extend the result to the case where the state is partially measured. As no separation principle can be invoked in the switched context, this extend is not trivial. Indeed, the convergence of the state estimation error to zero is dependent on the applied switching law. So, its design must guarantee both observation and control goals, whatever the values of the unknown estimation error. Thus, a direct application of the results in [21] is impossible since it implies that the estimation error must be known.

In Section 2, the problem statement is given and we recall the main result of paper [21]. This allows to explain to the reader why the provided feedback law not only stabilizes the switched system but also approaches the optimal solution.

In Section 3, assuming that there exists at least a controllable and observable convex combination of the subsystems and as the positive definite solution of an algebraic Riccati equation is a continuous function with respect to the constant matrices related to the equation, we are able to build a parametrized family of positive definite function whose parameters belong to a compact set. A control Lyapunov function is then defined as the point-wise infimum of this family.

We prove that the Lyapunov function is locally lipschitzian and homogenous of degree two. Then, we show that its directional Dini derivative is well defined along trajectories and we deduce an output feedback making the system semi global exponentially stable.

In section 4, asymptotic disturbance rejection is analysis and an adaptive observer is introduced that makes robust the design procedure with respect to noise as it is shown though an illustrative example in section 5 . Finally, in section 6 , we show that the design procedure can be applied to the dc-dc power converters.

\section{Problem statement and preliminary results}

We consider the class of continuous time linear switched systems:

$$
\left\{\begin{aligned}
\dot{x}(t) & =A_{\sigma(t)} x(t)+B_{\sigma(t)} u_{\sigma(t)}(t) \\
y & =C_{\sigma(t)} x
\end{aligned}\right.
$$

where $\sigma:[0,+\infty) \rightarrow \mathfrak{S}=\{1, \cdots, s\}$ denotes the switching law that selects the active mode at time $t$ by choosing among a finite collection of linear systems defined by the pairs $\left(A_{i}, B_{i}\right) \in \mathbf{R}^{n \times n} \times \mathbf{R}^{n \times m_{i}}, i \in \mathfrak{S}$. Each subsystem is also governed by a control $u_{i}(t) \in \mathbf{R}^{m_{i}}, 0 \leq m_{i} \leq n$ and has an output $x \mapsto C_{i} x$.

In [21] and in order to meet some performance requirements, we designed a state feedback switching law (i.e. $\left.x \mapsto\left(\sigma(x), u_{\sigma(x)}(x)\right)\right)$ for system (1a) that approaches the optimal solution of the following optimization problem:

Problem 1: Minimize the switched quadratic criterion:

$$
\min _{\sigma, u_{\sigma}} \frac{1}{2} \int_{0}^{\infty} x^{\mathrm{T}}(t) Q_{\sigma(t)} x(t)+u_{\sigma(t)}^{\mathrm{T}}(t) R_{\sigma(t)} u_{\sigma(t)}(t) \mathrm{d} t
$$

where the matrices $Q_{i}$ and $R_{i}$ are symmetric positive definite for every $i \in \mathfrak{S}$ and $x(t)$ is subject to $\dot{x}(t)=$ $A_{\sigma(t)} x(t)+B_{\sigma(t)} u_{\sigma(t)}(t), x(0)=x_{0}$.

More precisely, the provided feedback law is deduced from a relaxed version of Problem 1 given by:

Problem 2: Minimize the quadratic criterion:

$$
\min _{\lambda, u} \frac{1}{2} \int_{0}^{\infty} x^{T}(t) Q(\lambda(t)) x(t)+u^{T}(t) R(\lambda) u(t) \mathrm{d} t
$$

where $x(t)$ is subject to $\dot{x}=A(\lambda) x+B(\lambda) u, x(0)=x_{0}$. Here we denoted by $(u, \lambda)$ the control defined as

$$
u=\left(u_{1}, u_{2}, \cdots, u_{s}\right), \quad \lambda \in \Lambda
$$

where $\Lambda$ is the simplex defined as $\Lambda=\left\{\lambda \in \mathbf{R}^{s} \mid \sum_{i=1}^{s} \lambda_{i}=\right.$ 1 and $\left.\lambda_{i} \geq 0\right\}$; the matrices $A(\lambda), B(\lambda), Q(\lambda)$ and $R(\lambda)$ being defined as

$$
\begin{aligned}
& A(\lambda)=\sum_{i \in S} \lambda_{i} A_{i}, \quad B(\lambda)=\left[\lambda_{1} B_{1}, \ldots, \lambda_{s} B_{s}\right] \\
& Q(\lambda)=\sum_{i \in S} \lambda_{i} Q_{i}, \quad R(\lambda)=\operatorname{diag}\left(\lambda_{1} R_{1}, \ldots, \lambda_{s} R_{s}\right) .
\end{aligned}
$$

It can be noticed that Problem 1 is embedded into Problem 2 which is simply obtained by taking the convex hull of the vector fields and of the costs. Actually, solutions of problem 2 which are admissible for Problem 1, are those for which the value $\lambda(t)$ is located at the vertices of the simplex $\Lambda$ for all $t$. If not, control $\lambda(\cdot)$ is singular and Problem 1 does not admit an optimal solution $[19,3]$. Nevertheless, suboptimal solutions can always be achieved in these cases by chattering. This last point is due to the fact that the set of trajectories related to the switched system is dense into the set of trajectories of its relaxed version [14]. Thus, in any case, it is more convenient to solve Problem 2 and then after to deduce optimal or suboptimal solution for Problem 1. 
Up to now the exact solution of Problem 2 (or 1 ) is not available. Therefore, the provided design is to define a control Lyapunov function as a tight upper bound on the value function (the optimal cost). We mean tight in the sense that the two functions may coincide at some points.

In order to explain this last point, let us outline the design proposed in [21]. First, let us consider the following Riccati equation:

$$
A(\lambda)^{\mathrm{T}} P_{\lambda}+P_{\lambda} A(\lambda)-P_{\lambda}\left(\sum_{i=1}^{s} \lambda_{i} B_{i} R_{i}^{-1} B_{i}^{\mathrm{T}}\right) P_{\lambda}+Q(\lambda)=0
$$

It can be noticed that the above parametrized Riccati equation corresponds to the LQ subproblem obtained for a fixed value of $\lambda \in \Lambda$. Moreover, as $Q(\lambda)>0$, a sufficient condition for the existence of a positive definite solution $P_{\lambda}$ is that the pair $(A(\lambda), B(\lambda))$ is stabilizable. So, the set $\Lambda^{+}$is defined as the set of $\lambda \in \Lambda$ such that the pair $(A(\lambda), B(\lambda))$ is stabilizable and the greatest eigenvalue of $P_{\lambda}$ is less than $\nu_{\max }$, an arbitrary large number. The set $\Lambda^{+}$is non empty and has a non empty interior if there exists a $s$-tuple $\lambda^{0}$ such that the pair $\left(A\left(\lambda^{0}\right), B\left(\lambda^{0}\right)\right)$ is controllable. The next ingredient is the following Lyapunov function

$$
V_{m}(x) \triangleq \inf _{\lambda \in \Lambda^{+}} x^{\mathrm{T}} P_{\lambda} x
$$

where $\Lambda^{+}$is the compact subset of $\Lambda$ defined above.

The important point for our purpose is that the following theorem occurs:

Theorem 1 ([21]) Assume that the matrices $Q_{i}$ are positive definite and there exists at least a $\lambda^{0} \in \Lambda$ such that the pair $\left(A\left(\lambda^{0}\right), B\left(\lambda^{0}\right)\right)$ is controllable. For every $x \in \mathbf{R}^{n}$, we choose $\lambda(x) \in \ell(x)$ where $\ell(x)$ denotes the set of $\lambda \in \Lambda^{+}$such that $V_{m}(x)=x^{\mathrm{T}} P_{\lambda} x$ and

$$
i(x) \in \underset{i \in \mathfrak{S}}{\arg \min }\left(2 x^{\mathrm{T}} A_{K_{i, \lambda}^{\mathrm{T}}}^{\mathrm{T}} P_{\lambda} x+x^{\mathrm{T}} M_{i, \lambda} x\right),
$$

where $A_{K_{i, \lambda}}=A_{i}-B_{i} K_{i, \lambda}, K_{i, \lambda}=R_{i}^{-1} B_{i}^{T} P_{\lambda}$ and $M_{i, \lambda}=Q_{i}+K_{i, \lambda}^{T} R_{i} K_{i, \lambda}$.

Then the feedback $\sigma(x)=i(x)$ and $u(x)=-K_{i, \lambda} x$ stabilizes system (1a) with a cost no greater than $\frac{1}{2} V_{m}\left(x_{0}\right)$.

PROOF. The proof of this theorem uses the Lyapunov function $V_{m}$ defined above. Before entering into the proof of the theorem, we recall that, to ensure the asymptotic stability of a dynamical system, the function $t \mapsto$ $V_{m}(x(t))$ does not need to be derivable, it suffices that one of the Dini derivatives be negative (see e.g. [2]).
Given a function $\varphi$ defined on $[0,+\infty)$, recall that the Dini derivatives of $\varphi$ at $t$ are the four limits related to the difference quotient

$$
\mathcal{R}(t, h) \triangleq \frac{\varphi(t+h)-\varphi(t)}{h}
$$

namely

$$
\begin{aligned}
\overline{\mathrm{D}}^{+} \varphi(t) & =\varlimsup_{h \uparrow 0} \mathcal{R}(t, h) & \underline{\mathrm{D}}^{+} \varphi(t) & =\varlimsup_{h \uparrow 0} \mathcal{R}(t, h) \\
\overline{\mathrm{D}}^{-} \varphi(t) & =\varlimsup_{h \downarrow 0} \mathcal{R}(t, h) & \underline{\mathrm{D}}^{-} \varphi(t) & =\varliminf_{h \downarrow 0} \mathcal{R}(t, h) .
\end{aligned}
$$

Consider now a function $f$ defined on $\mathbf{R}^{n}$ and let $d$ be a vector of $\mathbf{R}^{n}$, as in [10] we shall denote by $f^{\prime}(x ; d)$ the following limit (if it exists)

$$
f^{\prime}(x ; d) \triangleq \lim _{\substack{h \rightarrow 0 \\ h>0}} \frac{f(x+h d)-f(x)}{h} .
$$

In order to prove the existence and to compute $V_{m}^{\prime}(x ; d)$, we use Theorem 6.1 in [10] whose conditions of application are clearly met here; thus $V_{m}^{\prime}$ exists and we have

$$
V_{m}^{\prime}(x ; d,)=\inf _{\lambda \in \ell(x)} v_{\lambda}^{\prime}(x ; d)
$$

where $v_{\lambda}(x)=x^{\mathrm{T}} P_{\lambda} x$. As the function $x \mapsto v_{\lambda}(x)$ is smooth, from (3) and from the expression of the derivative of $v_{\lambda}$, we infer that

$$
V_{m}^{\prime}(x ; d)=2 \inf _{\lambda \in \ell(x)}\left(d^{\mathrm{T}} P_{\lambda} x\right)
$$

Now a solution $x(t)$ of system (1a) in closed loop with the feedback defined in the statement of Th. 1 is absolutely continuous and so has a derivative, demoted by $\dot{x}(t)$, almost everywhere. This fact and the existence of $V_{m}^{\prime}$ imply that the function $t \mapsto V(x(t))$ is right differentiable almost everywhere and that its right derivative, denote by $\dot{V}(x)$, is given by the formula

$$
\dot{V}(x)=V_{m}^{\prime}(x ; \dot{x}) \text {. }
$$

We shall compute an upper bound of the derivative of $V_{m}$ along the trajectories of system (1a) in closed-loop with the feedback introduced in the theorem. First notice that if $i(x)$ and $\lambda(x)$ are chosen as in the theorem, we 
have $2 x^{\mathrm{T}} A_{K_{i, \lambda}} P_{\lambda} x \leq-x^{\mathrm{T}} M_{i, \lambda} x$. Now, we have

$$
\begin{aligned}
& \dot{V}_{m}(x)=V_{m}^{\prime}\left(x ; A_{K_{i(x), \lambda(x)}} x\right) \\
&=2 \min _{\lambda \in \ell(x)} x^{\mathrm{T}} A_{K_{i(x), \lambda(x)}^{\mathrm{T}}}^{\mathrm{T}} P_{\lambda} x \quad \text { from }(4) \\
& \leq 2 x^{\mathrm{T}} A_{K_{i(x), \lambda(x)}^{\mathrm{T}}}^{\mathrm{T}} P_{\lambda(x)} x \\
& \leq-x^{\mathrm{T}} M_{i(x), \lambda(x)} x \\
& \quad \text { from the definition of }(i(x), \lambda(x)) \\
& \leq-\frac{\eta_{0}}{\alpha_{1}} V_{m}(x) .
\end{aligned}
$$

Here $\beta_{0}$ and $\alpha_{1}$ are defined as

$$
\eta_{0}=\min _{i \in \mathfrak{S}} \inf _{x \in S^{n-1}} \inf _{\lambda \in \ell(x)} x^{T} M_{i, \lambda} x, \quad \alpha_{1}=\max _{x \in S^{n-1}} V_{m}(x)
$$

This inequality implies that

$$
V_{m}(x(t)) \leq e^{-\beta t} V_{m}\left(x_{0}\right)
$$

with $\beta=\frac{\eta_{0}}{\alpha_{1}}$. As $V_{m}$ is homogeneous of degree 2 , this last inequality, implies the global exponential stability. The upper bound on the cost $\left(1 / 2 V_{m}\left(x_{0}\right)\right)$ comes from the fact that

$$
\begin{gathered}
x^{\mathrm{T}} Q_{i(x)} x+x^{\mathrm{T}} K_{i(x), \lambda(x)}^{\mathrm{T}} R_{i(x)} K_{i(x), \lambda(x)} x= \\
x^{\mathrm{T}} M_{i(x), \lambda(x)} x \leq-\dot{V}_{m}(x) .
\end{gathered}
$$

Now we are able to discuss why the proposed switched law could be optimal and why $V_{m}$ can coincide with the value function at some points. One can observe first that for a given initial state $x_{0}$, the value $\frac{1}{2} V_{m}\left(x_{0}\right)$ is the best cost related to every constant convex combination $\lambda$ that stabilizes the relaxed system (i.e. such that the pair $(A(\lambda), B(\lambda))$ is stabilizable). In particular, if at least one subsystem $\left(A_{i}, B_{i}\right)$ (for a given $i \in \mathfrak{S}$ ) is stabilizable, then $\frac{1}{2} V_{m}(x) \leq \frac{1}{2} x^{T} P_{i} x$ where $P_{i}$ is the solution of the Riccati equation related to mode $i$. This point shows that the proposed switching rule is always consistent as defined in [13].

Actually, $V_{m}(x)$ is equal to the optimal cost along the part of trajectories where the optimal control $\lambda^{*}$ is constant to reach the origin. This situation occurs when:

- the number of switches is finite;

- the trajectory is steered to the origin by a constant singular control (we mean by singular control a control $\lambda$ which is not located at the vertices of the simplex $\Lambda$ ) for which $P_{\lambda}>0$. Excepted in degenerated case, it can be shown by a simple algebraic manipulation that in the two-dimensional case, singular controls are constant.

Formally, we can also justify the design of the switching law as follow. For this problem stated in infinite time, the necessary conditions provided by Pontryagin Maximum Principle (PMP) can be summarized by the following statement:

Theorem 2 ([21]) Suppose that $\left(\lambda^{*}, u^{*}\right)$ is optimal with the corresponding state $x^{*}$. Then, there exists an absolutely continuous function $p^{*}$, named co-state, such that:

(1) $p^{*} \not \equiv 0$

(2) $\dot{p}^{*}=-A(\lambda)^{T} p^{*}-Q(\lambda) x^{*}$ for almost all $t \in \mathbb{R}^{+}$,

(3) $u_{i}^{*}(t)=-R_{i}^{-1} B_{i}^{T} p^{*}(t)$,

(4) $\lambda^{*}(t)=\arg \min _{\lambda \in \Lambda} \sum_{i \in \mathfrak{S}} \lambda_{i} \mathcal{H}_{i}\left(x^{*},-R_{i}^{-1} B_{i}^{T} p^{*}, p^{*}\right)$ with $\mathcal{H}_{i}\left(x, u_{i}, p\right)=p^{T}\left(A_{i} x+B_{i} u_{i}\right)+\frac{1}{2}\left(x^{T} Q_{i} x+\right.$ $\left.u_{i}^{T} R_{i} u_{i}\right)$

Assuming known the value function denoted by $V^{*}(x)$, one can write from an initial condition $x_{0}$, for any $T>0$,

$$
\begin{array}{r}
V^{*}\left(x_{0}\right)=\min _{\sigma} \frac{1}{2} \int_{0}^{T} x^{T} Q_{\sigma(t)} x+u_{\sigma(t)}^{T} R_{\sigma(t)} u_{\sigma(t)} \mathrm{d} t \\
+V^{*}(x(T)) .
\end{array}
$$

The transversality condition of PMP implies that, at time $T, p^{*}(T)=\frac{\partial V^{*}(x(T))}{\partial x}$ (if exists). Now, assuming that $V^{*}(x(T))$ is approximated by $V_{m}(x(T))$, an approximation of $p^{*}(T)$ is given by $p^{*}(x(T)) \approx P_{\lambda} x(T)$ with $\lambda \in \ell(x)$. Thus, it is easy to check, using $P_{\lambda} x(T)$ instead of $p^{*}(x(T))$, that the minimization of the Hamiltonian at time $T$ (see item (3) and (4) in Theorem 2) leads to the provided feedback law defined in Theorem 1. As the problem is homogenous and if the approximation is "good", one can infer that $p^{*}(x) \approx P_{\lambda(x)} x$ with $\lambda(x) \in \ell(x)$ for every $x$. Roughly speaking, the state feedback switching law matches the optimal one when $P_{\lambda(x)} x$ is a good approximation of $p^{*}$. This is shown throughout an illustrative example at the end of the paper.

The main result in [21], is the design of a sub-optimal feedback law which exponentially stabilizes system (1a); the mode and the gain matrix are chosen in order to minimize the derivative of a Lyapunov function along the trajectories of the system. In [21], the state $x$ is assumed to be perfectly known and the feedback depends on $x$. In this paper, our aim is to prove that the same goal can be reached through an output feedback law; that is to say, we assume that the state is only partially measured ( $c f$. Eq. (1b)) and we want to design a feedback law in the same spirit as in [21] but which depends only on the observation $y$. To this end, as explained hereafter, we have to design an auxiliary dynamical system in order to get an estimate of the state of the switched system. In the sequel, we denote by $u$ the $s$-tuple $u=\left(u_{1}, u_{2}, \cdots, u_{s}\right)$ and we add the following notations to the ones intro- 
duced after the statement of Problem 2:

$$
\begin{aligned}
C(\lambda) & =\left[\lambda_{1} C_{1}^{\mathrm{T}}\left|\lambda_{2} C_{2}^{\mathrm{T}},\right| \cdots \mid \lambda_{s} C_{s}^{\mathrm{T}}\right]^{\mathrm{T}} \\
S(\lambda) & =\sum_{i \in \mathfrak{S}} \lambda_{i} S_{i}, \quad T=\operatorname{diag}\left(T_{1}, T_{2}, \cdots, T_{s}\right) .
\end{aligned}
$$

\section{Lyapunov based output switching law}

We consider the following system constituted by two coupled Riccati equations:

$$
\left\{\begin{array}{l}
A(\lambda)^{\mathrm{T}} P_{\lambda}+P_{\lambda} A(\lambda) \\
\quad-P_{\lambda}\left(\sum_{i=1}^{s} \lambda_{i} B_{i} R_{i}^{-1} B_{i}^{\mathrm{T}}\right) P_{\lambda}+Q(\lambda)=0 \\
A(\lambda) \Pi_{\lambda}+\Pi_{\lambda} A(\lambda)^{\mathrm{T}} \\
-\Pi_{\lambda}\left(\sum_{i=1}^{s} \lambda_{i} C_{i}^{\mathrm{T}} T_{i}^{-1} C_{i}\right) \Pi_{\lambda}+S(\lambda)=0 .
\end{array}\right.
$$

Notice that the matrices $\sum_{i=1}^{s} \lambda_{i} B_{i} R_{i}^{-1} B_{i}{ }^{\mathrm{T}}$ and $\sum_{i=1}^{s} \lambda_{i} C_{i}^{\mathrm{T}} T_{i}^{-1} C_{i}$ can be put under the form $\mathcal{B}_{\lambda} R^{-1} \mathcal{B}_{\lambda}^{\mathrm{T}}$ and $\mathcal{C}_{\lambda}^{\mathrm{T}} T^{-1} \mathcal{C}_{\lambda}$ respectively by letting

$$
\begin{aligned}
\mathcal{B}_{\lambda} & =\left[\sqrt{\lambda_{1}} B_{1}, \sqrt{\lambda_{2}} B_{2}, \cdots, \sqrt{\lambda_{s}} B_{s}\right] \\
\mathcal{C}_{\lambda} & =\left[\sqrt{\lambda_{1}} C_{1}^{\mathrm{T}}\left|\sqrt{\lambda_{2}} C_{2}^{\mathrm{T}}\right| \cdots \mid \sqrt{\lambda_{s}} C_{s}^{\mathrm{T}}\right]^{\mathrm{T}} \\
R & =\operatorname{diag}\left(R_{1}, \ldots, R_{s}\right), \quad T=\operatorname{diag}\left(T_{1}, \ldots, T_{s}\right)
\end{aligned}
$$

Now, using the Hautus test, it is quite obvious that the pair $\left(A(\lambda), \mathcal{B}_{\lambda}\right)$ is stabilizable (resp. controllable) if and only if the pair $(A(\lambda), B(\lambda))$ is stabilizable (resp. controllable); an analogous result concerning the detectability (resp. the observability) of the pair $\left(A, \mathcal{C}_{\lambda}\right)$ is true. If there exists $\lambda^{0} \in \Lambda$ such that the pair $\left(A\left(\lambda^{0}\right), B\left(\lambda^{0}\right)\right)$ is controllable and the pair $\left(A\left(\lambda^{0}\right), C\left(\lambda^{0}\right)\right)$ is observable, the same is true if $\lambda$ belongs to a sufficiently small neighborhood of $\lambda^{0}$ and so, in this case, the Riccati equations (5) admit a unique (positive definite) pair of solutions $\left(P_{\lambda}, \Pi_{\lambda}\right)$ for every $\lambda$ in some neighborhood of $\lambda^{0}$. Moreover, it is well known that the positive definite solution of a Riccati equation depends continuously on the parameters of the equation [16] and so $P_{\lambda}$ and $\Pi_{\lambda}$ are continuous functions of $\lambda$. Notice also that the condition for the existence of a symmetric nonnegative solution of the Riccati equations (5) can be weakened: in [15], $\mathrm{V}$. Kučera proved that if the pair $\left(A(\lambda), \mathcal{B}_{\lambda}\right)$ is stabilizable and if the matrix

$$
\mathcal{M}=\left(\begin{array}{cc}
A(\lambda) & -\mathcal{B}_{\lambda} R^{-1} \mathcal{B}_{\lambda}^{\mathrm{T}} \\
-Q(\lambda) & -A(\lambda)^{\mathrm{T}}
\end{array}\right)
$$

has no purely imaginary eigenvalues, then there exists a symmetric nonnegative solution to equation (5a). This result allow us to prove the following lemma about system (5).

Lemma 1 If the pair $(A(\lambda), B(\lambda))$ is stabilizable, the pair $(A(\lambda), C(\lambda))$ is detectable, and $Q(\lambda)$ and $S(\lambda)$ are positive definite, then there exists a unique pair of positive definite solutions to system (5).

PROOF. We prove only the existence of a positive definite solution to equation (5a), the existence of a positive definite solution to (5b) being similar. We denote by $\mathcal{M}_{1,2}$ the matrix $\mathcal{B}_{\lambda} R^{-1} \mathcal{B}_{\lambda}^{\mathrm{T}}$ and we take $x=\left(x_{1}^{\mathrm{T}}, x_{2}^{\mathrm{T}}\right)^{\mathrm{T}}$ a vector of $\mathbf{C}^{2 n}$ such that $\mathcal{M} x=i \alpha x$ (with $\alpha \in \mathbf{R}$ ); we shall see that $x=0$. We have

$$
\begin{aligned}
A(\lambda) x_{1}-\mathcal{M}_{1,2} x_{2} & =i \alpha x_{1} \\
-Q(\lambda) x_{1}-A(\lambda)^{\mathrm{T}} x_{2} & =i \alpha x_{2} .
\end{aligned}
$$

Multiplying on the left the members of equation (6) (resp. equation (7)) by $x_{2}^{*}$ (resp. by $x_{1}^{*}$ ), (the star denoting the conjugate transpose), we get

$$
\begin{aligned}
x_{2}^{*} A(\lambda) x_{1}-x_{2}^{*} \mathcal{M}_{1,2} x_{2} & =i \alpha x_{2}^{*} x_{1} \\
-x_{1}^{*} Q(\lambda) x_{1}-x_{1}^{*} A(\lambda)^{\mathrm{T}} x_{2} & =i \alpha x_{1}^{*} x_{2} .
\end{aligned}
$$

Now we add the complex conjugate of the members of equation (8) to the corresponding members of equation (9), taking into account that all the matrices involved in these equations are with real coefficients, we get

$$
-x_{1}^{*} Q(\lambda) x_{1}-x_{2}^{*} \mathcal{M}_{1,2} x_{2}=0 .
$$

If $x_{1} \neq 0$, as $Q(\lambda)$ is positive definite, this last equality implies $x_{2}^{*} \mathcal{M}_{1,2} x_{2}<0$, but this inequality cannot occur because $\mathcal{M}_{1,2}$ is nonnegative, so we must have $x_{1}=0$; reporting this equality in (6) and (7), we get $\mathcal{M}_{1,2} x_{2}=0$ and $A(\lambda)^{\mathrm{T}} x_{2}=-i \alpha x_{2}$, which in turn implies

$$
A(\lambda)^{\mathrm{T}} x_{2}=-i \alpha x_{2} \quad \text { and } \quad \mathcal{B}_{\lambda}^{\mathrm{T}} x_{2}=0
$$

because $R$ is positive definite. As the pair $(A(\lambda), B(\lambda))$ is stabilizable, so is the pair $\left(A(\lambda), \mathcal{B}_{\lambda}\right)$, therefore the pair $\left(A(\lambda)^{\mathrm{T}}, \mathcal{B}_{\lambda}^{\mathrm{T}}\right)$ is detectable. From this property and equalities $(10)$, it follows that $x_{2}=0$ by application of the Hautus lemma.

By application of the above-mentioned result from Kučera, we deduce that there exists a symmetric nonnegative solution $P_{\lambda}$ to equation (5a). Now this solution is necessarily definite; assume indeed that $v$ is a vector of $\mathbf{R}^{n}$ such that $P_{\lambda} v=0$, left-multiply both sides of (5a) by $v^{\mathrm{T}}$ and right-multiply by $v$, we get $v^{\mathrm{T}} Q(\lambda) v=0$ which implies $v=0$ since $Q(\lambda)$ is positive definite. 
We modify slightly the definition of the set $\Lambda^{+}$; from now on $\Lambda^{+}$will denote the set of $\lambda \in \Lambda$ such the pair $(A(\lambda), B(\lambda))$ is stabilizable, the pair $(A(\lambda), C(\lambda))$ is detectable and the eigenvalues of $P_{\lambda}$ and $\Pi_{\lambda}$ are less than $\nu_{\max }$, an arbitrary large number. This set $\Lambda^{+}$satisfies the following property.

Lemma 2 The matrices $Q_{i}$ and $S_{i}$ being positive definite, if there exists $\lambda^{0} \in \Lambda$ such that the pair $\left(A\left(\lambda^{0}\right), B\left(\lambda^{0}\right)\right)$ is controllable and the pair $\left(A\left(\lambda^{0}\right), C\left(\lambda^{0}\right)\right)$ is observable, then, for every $\nu_{\max }$ large enough, set $\Lambda^{+}$ is compact and its interior is not empty in $\Lambda$. Moreover, there exist positive real numbers $\alpha_{m}$ and $\alpha_{M}$ defined as

$$
\begin{aligned}
\alpha_{m} & =\min _{\lambda \in \Lambda^{+}} \min \left(\operatorname{spec}\left(P_{\lambda}\right) \cup \operatorname{spec}\left(\Pi_{\lambda}\right)\right), \\
\alpha_{M} & =\max _{\lambda \in \Lambda^{+}} \max \left(\operatorname{spec}\left(P_{\lambda}\right) \cup \operatorname{spec}\left(\Pi_{\lambda}\right)\right) .
\end{aligned}
$$

Here spec denotes the spectrum of a matrix.

PROOF. As noticed above, we can find a compact neighborhood $\mathcal{U}$ of $\lambda^{0}$ such that the Riccati equation (5) admits positive definite pair of solutions $\left(P_{\lambda}, \Pi_{\lambda}\right)$ for every $\lambda \in \mathcal{U}$. The mapping $\lambda \mapsto\left(P_{\lambda}, \Pi_{\lambda}\right)$ being continuous and $\mathcal{U}$ being compact, we have $\sup _{\lambda \in \mathcal{U}} \max \left(\operatorname{spec}\left(P_{\lambda}\right) \cup\right.$ $\left.\operatorname{spec}\left(\Pi_{\lambda}\right)\right)<\infty$; this implies that, if $\nu_{\max }$ is chosen large enough, the interior of $\Lambda^{+}$is non empty.

Notice that the hypothesis of the lemma imply obviously that $Q(\lambda)$ and $S(\lambda)$ are positive definite for every $\lambda \in \Lambda$. Now, set $\Lambda^{+}$is included in $\Lambda$, therefore it is bounded; we shall show that it is also closed. Assume that there exists a sequence $\left(\lambda^{k}\right)_{k \geq 1} \in \Lambda^{+}$such that $\lim _{k \rightarrow \infty} \lambda^{k}=\tilde{\lambda}$; as $\Lambda$ is a closed set, $\tilde{\lambda} \in \Lambda$. Moreover, the sequence $\left(\left(P_{\lambda^{k}}, \Pi_{\lambda^{k}}\right)\right)_{k \geq 1}$ is bounded, so we can assume that it converges to a pair of symmetric matrices $(\tilde{P}, \tilde{\Pi})$. As a limit of a sequence of pairs of positive definite matrices, $\tilde{P}$ and $\tilde{\Pi}$ are positive (semi) definite; moreover, for continuity reasons, they are solutions of (5) with $\lambda=\tilde{\lambda}$. We claim first that $\tilde{P}$ is definite, assume indeed that $v$ is a vector such that $\tilde{P} v=0$, left-multiply both sides of (5a) by $v^{\mathrm{T}}$ and right-multiply by $v$, we get $v^{\mathrm{T}} Q(\tilde{\lambda}) v=0$ which implies $v=0$ since $Q(\tilde{\lambda})$ is positive definite. Moreover the pair $(A(\tilde{\lambda}), B(\tilde{\lambda}))$ is stabilizable, to see this let $\mu \in \mathbf{C}$ be an eigenvalue of $A(\tilde{\lambda})^{\mathrm{T}}$ such that $\Re(\mu) \geq 0\left(\Re(\cdot)\right.$ stands for the real part) and let $v \in \mathbf{C}^{n}$ be a vector such that $A(\tilde{\lambda})^{\mathrm{T}} v=\mu v$ and $B^{\mathrm{T}}(\tilde{\lambda}) v=0$ (which implies that $\lambda_{i} B_{i}^{\mathrm{T}} v=0$ for $i=1, \ldots, s$ ), we shall see that $v=0$, which implies that the rank of the ma$\operatorname{trix}(A(\tilde{\lambda})-\mu \operatorname{Id}, B(\tilde{\lambda}))^{\mathrm{T}}$ is equal to $n$ for every $\mu$ in the closed right half plane and so the result will follow from the Hautus lemma. Matrix $\tilde{P}$ being definite, there exists $x \in \mathbf{C}^{n}$ such that $\tilde{P} x=v$. Left-multiply both sides of (5a) by $x^{*}$ and right-multiply by $x$, taking into account the above equalities, we get

$$
\mu x^{*} v+\bar{\mu} v^{*} x+x^{*} Q(\tilde{\lambda}) x=0 .
$$

If $x \neq 0$, as the matrix $Q(\tilde{\lambda})$ is definite positive, this equality implies that $\Re\left(\mu x^{*} v\right)<0$ but $x^{*} v=x^{*} \tilde{P} x$ and so $x^{*} v>0$ because $\tilde{P}$ is positive definite. Thus we must have $\Re(\mu)<0$, which is a contradiction. So, we have $x=0$, which implies that $v=0$. Similar arguments show that $\tilde{\Pi}$ is positive definite and that the pair $(A(\tilde{\lambda}), C(\tilde{\lambda}))$ is detectable.

Finally, the existence of $\alpha_{m}$ and $\alpha_{M}$ follows from the compactness of $\Lambda^{+}$and the continuity of the mapping $\lambda \mapsto\left(P_{\lambda}, \Pi_{\lambda}\right)$.

We shall now re-write equations (5); to this end we introduce the following notations: we denote by $A_{K}(\lambda)$ and $A_{L}(\lambda)$ the matrices defined as:

$$
A_{K}(\lambda) \triangleq \sum_{i \in \mathfrak{S}} \lambda_{i} A_{K_{i, \lambda}}, \quad A_{L}(\lambda) \triangleq \sum_{i \in \mathfrak{S}} \lambda_{i} A_{L_{i, \lambda}}
$$

where

$$
\begin{aligned}
A_{K_{i, \lambda}} & \triangleq A_{i}-B_{i} K_{i, \lambda} & A_{L_{i, \lambda}} & \triangleq A_{i}-L_{i, \lambda} C_{i} \\
K_{i, \lambda} & \triangleq R_{i}^{-1} B_{i}^{T} P_{\lambda} & L_{i, \lambda} & \triangleq \Pi_{\lambda} C_{i}^{\mathrm{T}} T_{i}^{-1}
\end{aligned}
$$

and by $M(\lambda)$ and $N(\lambda)$ the matrices

$$
\begin{array}{rlrl}
M(\lambda) & \triangleq \sum_{i \in \mathfrak{S}} \lambda_{i} M_{i, \lambda} & N(\lambda) \triangleq \sum_{i \in \mathfrak{S}} \lambda_{i} N_{i, \lambda} \\
M_{i, \lambda} \triangleq Q_{i}+K_{i, \lambda}^{\mathrm{T}} R_{i} K_{i, \lambda} & N_{i, \lambda} \triangleq S_{i}+L_{i, \lambda} T_{i} L_{i, \lambda}^{\mathrm{T}}
\end{array}
$$

The Riccati equations (5) can then be rewritten as :

$$
\left\{\begin{array}{l}
A_{K}(\lambda)^{\mathrm{T}} P_{\lambda}+P_{\lambda} A_{K}(\lambda)+M(\lambda)=0 \\
A_{L}(\lambda) \Pi_{\lambda}+\Pi_{\lambda} A_{L}(\lambda)^{\mathrm{T}}+N(\lambda)=0
\end{array}\right.
$$

Lemma 3 Let $q$ be a positive number, for every $(z, E, \lambda) \in \mathbf{R}^{n} \times \mathbf{R}^{n \times n} \times \Lambda^{+}$, we have

$$
\begin{aligned}
& \min _{i \in \mathfrak{S}}\left(2 z^{\mathrm{T}} A_{K_{i, \lambda}^{\mathrm{T}}}^{\mathrm{T}} P_{\lambda} z+z^{\mathrm{T}} M_{i, \lambda} z\right. \\
& \left.+q \operatorname{Tr}\left(2 E^{\mathrm{T}} A_{L_{i, \lambda}}^{\mathrm{T}} \Pi_{\lambda}^{-1} E+E^{\mathrm{T}} \Pi_{\lambda}^{-1} N_{i, \lambda} \Pi_{\lambda}^{-1} E\right)\right) \leq 0 .
\end{aligned}
$$

here $\operatorname{Tr}$ denotes the trace of a matrix.

PROOF. Take $(z, E) \in \mathbf{R}^{n} \times \mathbf{R}^{n \times n}$ and $\lambda \in \Lambda^{+}$, then equation (13) admits a pair of positive definite matrices 
as (unique) solution; notice that equation (13b) can be rewritten as

$$
\Pi_{\lambda}^{-1} A_{L}(\lambda)+A_{L}(\lambda)^{\mathrm{T}} \Pi_{\lambda}^{-1}+\Pi_{\lambda}^{-1} N(\lambda) \Pi_{\lambda}^{-1}=0 .
$$

Multiply both sides of equation (13a) by $z^{\mathrm{T}}$ on the left and by $z$ on the right.Multiply also both sides of equation (14) by $E^{\mathrm{T}}$ on the left and by $E$ on the right, and take the trace of the obtained expression. By adding the two obtained equalities and letting

$$
\begin{aligned}
\mathcal{R}_{i}= & \lambda_{i}\left(2 z^{\mathrm{T}} A_{K_{i, \lambda}}^{\mathrm{T}} P_{\lambda} z+z^{\mathrm{T}} M_{i, \lambda} z\right. \\
& \left.+q \operatorname{Tr}\left(2 E^{\mathrm{T}} A_{L_{i, x l}}^{\mathrm{T}} \Pi_{\lambda}^{-1} E+E^{\mathrm{T}} \Pi_{\lambda}^{-1} N_{i, \lambda} \Pi_{\lambda}^{-1} E\right)\right)
\end{aligned}
$$

we clearly get:

$$
\sum_{i=1}^{s} \lambda_{i} \mathcal{R}_{i}=0
$$

so we cannot have $\lambda_{i} \mathcal{R}_{i}>0$ for every $i \in \mathfrak{S}$ because in this case the left-hand member of equality (15) would be positive. Thus, for every triple $(z, E, \lambda)$, there exists an index $i$ such that $\mathcal{R}_{i} \leq 0$, which implies the inequality of the lemma.

\subsection{A Lyapunov function}

Hereafter, we denote by $\|\cdot\|$ the usual euclidean norm on $\mathbf{R}^{n}$ and we use the same notation to denote the induced matricial norm on $\mathbf{R}^{n \times n}$. On $\mathbf{R}^{n \times n}$ we consider also the norm $\mathcal{N}_{\lambda}$ defined, for $\lambda \in \Lambda^{+}$, as $\mathcal{N}_{\lambda}(M)=$ $\left(\operatorname{Tr}\left(M^{\mathrm{T}} \Pi_{\lambda}^{-1} M\right)\right)^{1 / 2}$ where $\Pi_{\lambda}$ is the solution of $(13 \mathrm{~b})$. Using that the matrix $\Pi_{\lambda}^{-1}$ is diagonalizable, a direct calculation shows that

$$
\rho_{m}\left(\Pi_{\lambda}^{-1}\right)^{1 / 2}\|M\| \leq \mathcal{N}_{\lambda}(M) \leq n^{1 / 2} \rho_{M}\left(\Pi_{\lambda}^{-1}\right)^{1 / 2}\|M\|
$$

for every matrix $M \in \mathbf{R}^{n \times n}$, here $\rho_{m}$ (resp. $\rho_{M}$ ) denotes the least eigenvalue (resp. the greatest eigenvalue) of a matrix. Choose $q>0$, and let us now introduce the following function defined on $\mathbf{R}^{n} \times \mathbf{R}^{n \times n}$ :

$$
W_{m}(z, E) \triangleq \inf _{\lambda \in \Lambda^{+}}\left(z^{\mathrm{T}} P_{\lambda} z+q \operatorname{Tr}\left(E^{\mathrm{T}} \Pi_{\lambda}^{-1} E\right)\right)
$$

where $\left(P_{\lambda}, \Pi_{\lambda}\right)$ denotes the solution of equation (13). Clearly, as $P_{\lambda}$ and $\Pi_{\lambda}$ are positive definite when $\lambda$ belongs to $\Lambda^{+}$and as the set $\left\{\left(P_{\lambda}, \Pi_{\lambda}\right) \mid \lambda \in \Lambda^{+}\right\}$is compact, $W_{m}$ is a positive definite function; notice also that $W_{m}$ is homogeneous of degree 2 and, as we shall see, locally lipschitzian.

Proposition 4 Function $W_{m}$ defined by relation (17) is locally lipschitzian.
PROOF. We have $z^{\mathrm{T}} P_{\lambda} z \leq \alpha_{M}\|z\|^{2}$ for every $(z, \lambda) \in$ $\mathbf{R}^{n} \times \Lambda^{+}$. So, if we take $z_{1}$ and $z_{2}$ in the ball $B(0, R)$, we obtain easily that, for every $\lambda \in \Lambda^{+}$,

$$
\begin{aligned}
\left|z_{1}^{\mathrm{T}} P_{\lambda} z_{1}-z_{2}^{\mathrm{T}} P_{\lambda} z_{2}\right|=\left|\left(z_{1}-z_{2}\right)^{\mathrm{T}} P_{\lambda}\left(z_{1}+z_{2}\right)\right| & \\
& \leq K_{1}\left\|z_{1}-z_{2}\right\|
\end{aligned}
$$

where $K_{1}=2 \alpha_{M} R$. On the other hand, if the matrices $E_{1}$ and $E_{2}$ are in the ball $B(0, R)$, we have

$$
\begin{aligned}
\mid \operatorname{Tr}\left(E_{1}^{\mathrm{T}} \Pi_{\lambda}^{-1} E_{1}\right)- & \operatorname{Tr}\left(E_{2}^{\mathrm{T}} \Pi_{\lambda}^{-1} E_{2}\right) \mid \\
& =\left|\operatorname{Tr}\left(\left(E_{1}-E_{2}\right)^{\mathrm{T}} \Pi_{\lambda}^{-1}\left(E_{1}+E_{2}\right)\right)\right| \\
& \leq K_{2}\left\|E_{1}-E_{2}\right\|
\end{aligned}
$$

where $K_{2}=2 n \alpha_{m}^{-1} R$. Thus, the family of functions $\left(w_{\lambda}\right)_{\lambda \in \Lambda^{+}}$defined by

$$
w_{\lambda}(z, E)=z^{T} P_{\lambda} z+q \operatorname{Tr}\left(E^{\mathrm{T}} \Pi_{\lambda}^{-1} E\right)
$$

is uniformly (with respect to $\lambda$ ) locally liptchitzian with a Lipschitz constant equals to $K \triangleq \max \left(K_{1}, K_{2}\right)$ on the ball $B(0, R)$.

Now, as the function $w_{\lambda}$ is continuous and set $\Lambda^{+}$is compact, there exists a pair $\left(\lambda_{1}, \lambda_{2}\right) \in\left(\Lambda^{+}\right)^{2}$ such that:

$$
W_{m}\left(z_{1}, E_{1}\right)=w_{\lambda^{1}}\left(z_{1}, E_{1}\right) \quad W_{m}\left(z_{2}, E_{2}\right)=w_{\lambda^{2}}\left(z_{2}, E_{2}\right) .
$$

From the definition of $W_{m}$, we deduce easily that

$$
\begin{aligned}
& w_{\lambda^{1}}\left(z_{1}, E_{1}\right)=W_{m}\left(z_{1}, E_{1}\right) \leq w_{\lambda^{2}}\left(z_{1}, E_{1}\right) \\
& w_{\lambda^{1}}\left(z_{2}, E_{2}\right) \geq W_{m}\left(z_{2}, E_{2}\right)=w_{\lambda^{2}}\left(z_{2}, E_{2}\right)
\end{aligned}
$$

therefore, by continuity, there exists $(z, E)$ on the line segment $\left[\left(z_{1}, E_{1}\right)\left(z_{2}, E_{2}\right)\right]$ such that $w_{\lambda^{1}}(z, E)=$ $w_{\lambda^{2}}(z, E)$ and it follows:

$$
\begin{aligned}
& \left|W_{m}\left(z_{1}, E_{1}\right)-W_{m}\left(z_{2}, E_{2}\right)\right| \\
& \quad \leq\left|w_{\lambda_{1}}\left(z_{1}, E_{1}\right)-w_{\lambda_{1}}(z, E)\right| \\
& \quad+\left|w_{\lambda_{2}}(z, E)-w_{\lambda_{2}}\left(z_{2}, E_{2}\right)\right| \\
& \quad \leq K\left(\left\|z_{1}-z\right\|+\left\|E_{1}-E\right\|\right) \\
& \quad+K\left(\left\|z-z_{2}\right\|+\left\|E-E_{2}\right\|\right) \\
& \quad K\left(\left\|z_{1}-z_{2}\right\|+\left\|E_{1}-E_{2}\right\|\right)
\end{aligned}
$$

Finally we have $z^{\mathrm{T}} P_{\lambda} z \geq \rho_{m}\left(P_{\lambda}\right)\|z\|^{2}$ and $\operatorname{Tr}\left(E^{\mathrm{T}} \Pi_{\lambda}^{-1} E\right) \geq$ $\rho_{m}\left(\Pi_{\lambda}^{-1}\right)\|E\|^{2}(c f .(16))$ and so

$$
W_{m}(z, E) \geq K^{\prime}\left(\|z\|^{2}+\|E\|^{2}\right)
$$

where $K^{\prime}=\min \left(\alpha_{m}, 1 / \alpha_{M}\right)$. As, moreover, $W_{m}$ is continuous, it is proper. 
The derivative of $W_{m}$ As for function $V_{m}$, the conditions of application of Th. 6.1 in [10] are clearly met, so the differential of $W_{m}$ (in the sense of [10]) exists and we have

$$
W_{m}^{\prime}(z, E ; d, F)=\inf _{\lambda \in \ell(z, E)} w_{\lambda}^{\prime}(z, E ; d, F)
$$

Here $\ell(z, E)$ denotes the set of $\lambda \in \Lambda^{+}$such that $W_{m}(z, E)=w_{\lambda}(z, E)$ (this set is clearly nonempty and compact because $\Lambda^{+}$is compact and the function $\lambda \mapsto w_{\lambda}(z, E)$ is continuous). As the function $(z, E) \mapsto w_{\lambda}(z, E)$ is smooth, from (18) and from the expression of the derivative of $w_{\lambda}$, we infer that

$$
W_{m}^{\prime}(z, E ; d, F)=2 \inf _{\lambda \in \ell(z, E)}\left(d^{\mathrm{T}} P_{\lambda} z+q \operatorname{Tr}\left(F^{\mathrm{T}} \Pi_{\lambda}^{-1} E\right)\right) .
$$

\subsubsection{Output stabilization}

In the sequel, we shall consider mappings from $\mathbf{R}^{n} \times$ $\mathbf{R}^{n \times n}$ to $\mathfrak{S} \times \Lambda^{+}$of the form $(\hat{x}, E) \mapsto(i(\hat{x}, E), \lambda(\hat{x}, E))$ such that $\lambda(\hat{x}, E) \in \ell(\hat{x}, E)$. To such a mapping, we relate the following feedback law for system (1a): the mode $\sigma(t)$ is equal to $i(\hat{x}(t), E(t))$ for every $t \geq 0$ and $u_{\sigma(t)}$ is equal to $-K_{i(\hat{x}, E), \lambda(\hat{x}, E)} \hat{x}$.

We consider now the following system:

$$
\left\{\begin{array}{c}
\dot{\hat{x}}=A_{\sigma(t)} \hat{x}-B K_{\sigma(t), \lambda(t)} \hat{x} \\
-L_{\sigma(t), \lambda(t)}\left(C_{\sigma(t)} \hat{x}-y\right) \\
\dot{x}=A_{\sigma(t)} x-B K_{\sigma(t), \lambda(t)} \hat{x}
\end{array}\right.
$$

the matrices $K_{\sigma, \lambda}$ and $L_{\sigma, \lambda}$ being defined as in (12). The question is: is it possible to find a law $(\sigma, \lambda)$ in such a way that system (20) is globally asymptotically stable about the origin? We shall see that, indeed such a law exists, moreover this law will be a feedback which depends on $\hat{x}$ and some matrix to be defined later. Introducing the estimation error $e \triangleq \hat{x}-x$, system (20) can be re-written as

$$
\left\{\begin{array}{l}
\dot{\hat{x}}=A_{K_{\sigma(t), \lambda(t)}} \hat{x}-L_{\sigma(t), \lambda(t)} C_{\sigma(t)} e \\
\dot{e}=A_{L_{\sigma(t), \lambda(t)}} e
\end{array}\right.
$$

Now, we are faced to the following problem. In order to choose the mode $i$ and the parameter $\lambda$ which determines the gain matrices $K_{i, \lambda}$ and $L_{i, \lambda}$ at every time $t$, we cannot proceed as in the case where the whole state is measurable, that is to say we cannot choose the pair $(i, \lambda)$ as in Theorem 1 because we do not have access to all the components of $e$. To overcome this difficulty, the trick consists in considering a slightly different system than (21):

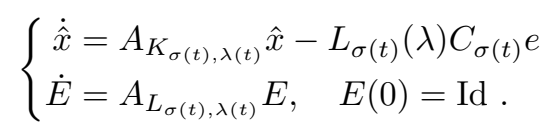

where $E$ evolves in $\mathbf{R}^{n \times n}$; notice that $e(t)=E(t) e(0)$; here Id stands for the $n$-dimensional identity matrix.

Theorem 3 Recall that the matrices $Q_{i}$ as well as the matrices $S_{i}$ are assumed to be positive definite. We assume that there exists at least one $s$-tuple $\lambda^{0} \in \Lambda$ such that the pair $\left(A\left(\lambda^{0}\right), B\left(\lambda^{0}\right)\right)$ is controllable and the pair $\left(A\left(\lambda^{0}\right), C\left(\lambda^{0}\right)\right)$ is observable and we consider the subset $\Lambda^{+}$of $\Lambda$ as defined in Lemma 2. For every $(\hat{x}, E) \in$ $\mathbf{R}^{n} \times \mathbf{R}^{n \times n}$, we choose

$$
\begin{gathered}
(i(\hat{x}, E), \lambda(\hat{x}, E)) \in \underset{(i, \lambda) \in \mathfrak{S} \times \ell(\hat{x}, E)}{\arg \min }\left(2 \hat{x}^{\mathrm{T}} A_{K_{i, \lambda}}^{\mathrm{T}} P_{\lambda} \hat{x}\right. \\
\left.+\hat{x}^{\mathrm{T}} M_{i, \lambda} \hat{x}+q \operatorname{Tr}\left(2 E^{\mathrm{T}} A_{L_{i, \lambda}}^{\mathrm{T}} \Pi_{\lambda}^{-1} E+E^{\mathrm{T}} \Pi_{\lambda}^{-1} N_{i, \lambda} \Pi_{\lambda}^{-1} E\right)\right) .
\end{gathered}
$$

Then the feedback related to $(i(\hat{x}, E), \lambda(\hat{x}, E))$ stabilizes system (22) locally exponentially provided that the norm of e $(0)$ is less than some constant $\eta(q)$; this constant tends to infinity as q tends to infinity.

Remark 5 From a practical point of view, Th. 3 remains valid if the feedback switching law is simplified as follows: for a given pair $(\hat{x}, E)$, choose $\lambda(\hat{x}, E)$ in $\ell(\hat{x}, E)$ and choose $i(\hat{x}, E)$ as follows

$$
\begin{aligned}
& i(\hat{x}, E) \in \underset{i \in \mathfrak{S}}{\arg \min }\left(2 \hat{x}^{\mathrm{T}} A_{K_{i, \hat{\lambda}}}^{\mathrm{T}} P_{\hat{\lambda}} \hat{x}+\hat{x}^{\mathrm{T}} M_{i, \hat{\lambda}} \hat{x}\right. \\
& \left.+q \operatorname{Tr}\left(2 E^{\mathrm{T}} A_{L_{i, \hat{\lambda}}}^{\mathrm{T}} \Pi_{\hat{\lambda}}^{-1} E+E^{\mathrm{T}} \Pi_{\hat{\lambda}}^{-1} N_{i, \hat{\lambda}} \Pi_{\hat{\lambda}}^{-1} E\right)\right)
\end{aligned}
$$

where $\hat{\lambda} \triangleq \lambda(\hat{x}, E)$.

The consequence of this theorem is that, with this feedback law, the state $(\hat{x}, e)$ of system $(21)$ is also (locally) exponentially stable about the origin, which means that the system (1a) in closed loop with this feedback law is exponentially stable about the origin, provided that $\hat{x}(0)$ is chosen in a ball of radius $\eta(q)$ centered at $x(0)$.

PROOF. [Proof of Th. 3] We shall compute the derivative of $W_{m}$ along the trajectories of system (21) in closedloop with the feedback introduced in the theorem. Hereafter, for the sake of readability, we denote by $\hat{\imath}$ and $\hat{\lambda}$ the index $i(\hat{x}, E)$ and the parameter $\lambda(\hat{x}, E)$ respectively. As in the proof of Th. 1, we can prove that the function $t \mapsto W_{m}(z(t), E(t))$ is right differentiable almost every- 
where and we have We have

$$
\begin{aligned}
\dot{W}_{m}(z(t), E(t))= & \left(W_{m}\right)^{\prime}\left(\hat{x}, E ; A_{K_{\hat{\imath}, \hat{\lambda}}} \hat{x}, A_{L_{\hat{\imath}, \hat{\lambda}}} E\right) \\
= & 2 \min _{\lambda \in \ell(\hat{x}, E)}\left(\hat{x}^{\mathrm{T}} A_{K_{\hat{\imath}, \hat{\lambda}}^{\mathrm{T}}}^{\mathrm{T}} P_{\lambda} \hat{x}-e^{\mathrm{T}} C_{\hat{\imath}}^{\mathrm{T}} L_{\hat{\imath}, \hat{\lambda}}^{\mathrm{T}} P_{\lambda} \hat{x}\right. \\
& \left.+q \operatorname{Tr}\left(E^{\mathrm{T}} A_{L_{\hat{\imath}, \hat{\lambda}}}^{\mathrm{T}} \Pi_{\lambda}^{-1} E\right)\right) \quad \text { from }(19) \\
\leq & 2\left(\hat{x}^{\mathrm{T}} A_{K_{\hat{\imath}, \hat{\lambda}}}^{\mathrm{T}} P_{\hat{\lambda}} \hat{x}-e^{\mathrm{T}} C_{\hat{\imath}}^{\mathrm{T}} L_{\hat{\imath}, \hat{\lambda}}^{\mathrm{T}} P_{\hat{\lambda}} \hat{x}\right. \\
& \left.+q \operatorname{Tr}\left(E^{\mathrm{T}} A_{L_{\hat{\imath}, \hat{\lambda}}^{\mathrm{T}}}^{\mathrm{T}} \Pi_{\hat{\lambda}}^{-1} E\right)\right)
\end{aligned}
$$

Now, from the definition of the pair $(i(\hat{x}, E), \lambda(\hat{x}, E))$ and by applying Lemma 3 , we see that the right-hand member in (23) is no greater than

$-\hat{x}^{\mathrm{T}} M_{\hat{\imath}, \hat{\lambda}}^{\mathrm{T}} \hat{x}-q \operatorname{Tr}\left(E^{\mathrm{T}} \Pi_{\hat{\lambda}}^{-1} N_{\hat{\imath}, \hat{\lambda}} \Pi_{\hat{\lambda}}^{-1} E\right)-2 e^{\mathrm{T}} C_{\hat{\imath}}^{\mathrm{T}} L_{\hat{\imath}, \hat{\lambda}}^{\mathrm{T}} P_{\hat{\lambda}} \hat{x}$,

clearly we have $M_{\hat{\imath} \hat{\lambda}} \leq Q_{\hat{\imath}}$ and $N_{\hat{\imath} \hat{\lambda}} \leq S_{\hat{\imath}}$, so from (23), we get

$$
\begin{aligned}
\dot{W}_{m}(z(t), E(t)) \leq-\hat{x}^{\mathrm{T}} Q_{\hat{\imath}}^{\mathrm{T}} \hat{x}- & q \operatorname{Tr}\left(E^{\mathrm{T}} \Pi_{\hat{\lambda}}^{-1} S_{\hat{\imath}} \Pi_{\hat{\lambda}}^{-1} E\right) \\
& -2 e^{\mathrm{T}} C_{\hat{\imath}}^{\mathrm{T}} L_{\hat{\imath}, \hat{\lambda}}^{\mathrm{T}} P_{\hat{\lambda}} \hat{x}
\end{aligned}
$$

In this inequality the first two terms between the parenthesis are clearly positive; from the assumptions of the theorem, $Q_{\hat{\imath}}$ is positive definite and this is also the case for the matrix $E^{\mathrm{T}} \Pi_{\hat{\lambda}}^{-1} S_{\hat{\imath}} \Pi_{\hat{\lambda}}^{-1} E$ (because $E$ is an invertible matrix) and so its trace is positive. We shall find an upper bound for the third term.

Hereafter, we introduce a family of norms on $\mathbf{R}^{n \times n}$ indexed by $(i, \lambda) \in \mathfrak{S} \times \Lambda^{+}$, they are defined as follows:

$$
\mathcal{N}_{i, \lambda}(M)=\left(\operatorname{Tr}\left(M^{\mathrm{T}} \Pi_{\lambda}^{-1} S_{i} \Pi_{\lambda}^{-1} M\right)\right)^{1 / 2}, \quad M \in \mathbf{R}^{n \times n}
$$

$\mathcal{N}_{i, \lambda}$ is clearly a norm defined on $\mathbf{R}^{n \times n}$ because the matrix $\Pi_{\lambda}^{-1} S_{i} \Pi_{\lambda}^{-1}$ is positive definite. Now $(i, \lambda)$ belongs to the set $\mathfrak{S} \times \Lambda^{+}$, which is compact, and the mapping $\lambda \mapsto \Pi_{\lambda}^{-1}$ is continuous, therefore the minimum

$$
\eta_{m}=\min \left\{\mathcal{N}_{i, \lambda}(E) \mid(i, \lambda) \in \mathfrak{S} \times \Lambda^{+} \text {and }\|E\|=1\right\}
$$

is positive. We have

$$
\left|e^{\mathrm{T}} C_{\hat{\imath}}^{\mathrm{T}} L_{\hat{\imath}, \hat{\lambda}}^{\mathrm{T}} P_{\hat{\lambda}} \hat{x}\right| \leq \beta_{1}\|e\|\|\hat{x}\| \leq \beta_{1}\|e(0)\|\|E\|\|\hat{x}\|
$$

where $\beta_{1}$ is an upper bound for the norms of the matrices $C_{i}^{\mathrm{T}} L_{i, \lambda}^{\mathrm{T}} P_{\lambda}$ when $(i, \lambda) \in \mathfrak{S} \times \Lambda^{+}$. From the norms equivalence, we know that there exists $k_{i, \lambda}$ such that

$$
\|E\| \leq k_{\hat{\imath}, \hat{\lambda}} \mathcal{N}_{\hat{\imath}, \hat{\lambda}}(E)
$$

the constant $k_{\hat{\imath}, \hat{\lambda}}$ can be taken equal to

$$
\frac{1}{k_{\hat{\imath}, \hat{\lambda}}}=\min _{\|F\|=1} \mathcal{N}_{\hat{\imath}, \hat{\lambda}}(F) ;
$$

obviously we have $\eta_{m} \leq 1 / k_{\hat{\imath}, \hat{\lambda}}$, and so

$$
\|E\| \leq \frac{1}{\eta_{m}} \mathcal{N}_{\hat{\imath}, \hat{\lambda}}(E) .
$$

On the other hand, there exists $\eta^{\prime}$ such that

$$
\|\hat{x}\| \leq \eta^{\prime}\left(\hat{x}^{\mathrm{T}} Q_{i} \hat{x}\right)^{1 / 2}
$$

for every $i \in \mathfrak{S}$. So, substituting the inequalities (27) and (28) in (25), we get

$$
\left|e^{\mathrm{T}} C_{\hat{\imath}}^{\mathrm{T}} L_{\hat{\imath}, \hat{\lambda}}^{\mathrm{T}} P_{\hat{\lambda}} \hat{x}\right| \leq \beta_{2}\|e(0)\|\left(\hat{x}^{\mathrm{T}} Q_{\hat{\imath}} \hat{x}\right)^{1 / 2} \mathcal{N}_{\hat{\imath}, \hat{\lambda}}(E)
$$

where $\beta_{2}=\beta_{1} \eta^{\prime} / \eta_{m}$.

Finally, reporting inequality (29) in (28), we obtain

$$
\begin{aligned}
& \dot{W}_{m}(x(t)) \leq \\
& -\hat{x}^{\mathrm{T}} Q_{\hat{\imath}} \hat{x}+2 \beta_{2}\|e(0)\|\left(\hat{x}^{\mathrm{T}} Q_{\hat{\imath}} \hat{x}\right)^{1 / 2} \mathcal{N}_{\hat{\imath}, \hat{\lambda}}(E)-q \mathcal{N}_{\hat{\imath}, \hat{\lambda}}(E)^{2} .
\end{aligned}
$$

Clearly, the expression in the right-hand member of this inequality is negative as soon as $\|e(0)\|<\eta(q) \triangleq \frac{\sqrt{q}}{\beta_{2}}$. To be more precise, if this inequality is satisfied, this right-hand member can be made less than a negative definite quadratic function of $(\hat{x}, E)$, which implies the exponential stability of system $(22)$.

\subsection{Perturbation analysis}

What happens in case of perturbation? Assume that this perturbation has the effect of re-initializing the state $x$ at some time $t_{k}$. So at this time $t_{k}$, we have $x\left(t_{k}\right)=$ $x\left(t_{k}^{-}\right)+\delta_{k}$, what is the consequence for the derivative of $W_{m}$ ? In inequality (24), we have to take into account this term $\delta_{k}$, so, denoting by $\rho(t)$, the right-hand member of this inequality, we have:

$$
\rho\left(t_{k}^{+}\right)=\rho\left(t_{k}\right)-2 \delta_{k}^{\mathrm{T}} C_{\hat{\imath}}^{\mathrm{T}} L_{\hat{\imath}, \hat{\lambda}}^{\mathrm{T}} P_{\hat{\lambda}} \hat{x}\left(t_{k}\right),
$$

here $\rho\left(t_{k}^{+}\right)=\lim _{h \uparrow 0}\left(\rho\left(t_{k}+h\right)\right.$. The term $\delta_{k}^{\mathrm{T}} C_{\hat{\imath}}^{\mathrm{T}} L_{\hat{\imath}, \hat{\lambda}}^{\mathrm{T}} P_{\hat{\lambda}} \hat{x}\left(t_{k}\right)$ is of degree one with respect to the pair $(\hat{x}, E)$, and so when $(\hat{x}, E)$ is small, it can be predominant so as $\rho(t)$ could become positive for some $t>t_{k}$.

We shall see how to modify the design of our observer in order to take into account the possible perturbations on the state $x$ and the observation $y$. We begin by noticing that the term $e^{\mathrm{T}} C_{\hat{\imath}}^{\mathrm{T}} L_{\hat{\imath}, \hat{\lambda}}^{\mathrm{T}} P_{\hat{\lambda}} \hat{x}$ in inequality (24) can be upper bounded as follows:

$$
e^{\mathrm{T}} C_{\hat{\imath}}^{\mathrm{T}} L_{\hat{\imath}, \hat{\lambda}}^{\mathrm{T}} P_{\hat{\lambda}} \hat{x} \leq \beta_{3}\left\|C_{\hat{\imath}} e\right\|\|\hat{x}\|
$$


where $\beta_{3}$ is an upper bound for the norms of the set of matrices $\left\{P_{\lambda} L_{i, \lambda} \mid(i, \lambda) \in \mathfrak{S} \times \Lambda^{+}\right\}$. So the expression in the right-hand member of (24) is negative as soon as

$$
\left\|C_{\hat{\imath}} e\right\|<\frac{\sqrt{q}}{\beta_{3} \eta^{\prime}} \mathcal{N}_{i, \lambda}(E)
$$

(recall that $\eta^{\prime}$ is a constant defined by inequality (28)).

The equations of the observer (22) are then modified as follows: equation (22a) is retained but equation (22b) is replaced by

$$
\dot{\mathcal{E}}(t)=\frac{\dot{G}}{G} \mathcal{E}+A_{L_{\sigma(t), \lambda}} \mathcal{E}, \quad \mathcal{E}(0)=\mathrm{Id}
$$

(recall that Id denotes the identity matrix), where the gain $G$ is subject to the following differential equation

$$
\dot{G}=-(1-\mu(t)) s_{1}(G-1)+\mu(t) s_{2} G, \quad G(0)=1 .
$$

Here $s_{1}$ and $s_{2}$ are constant positive parameters and $\mu$ is a continuous function defined as

$$
\mu(t)= \begin{cases}0 & \text { if }\left\|C_{\hat{\imath}} e(t)\right\| \leq \kappa_{1} \mathcal{N}_{\hat{\imath}, \hat{\lambda}}(\mathcal{E}) \\ \in(0,1) & \text { if } \kappa_{1} \mathcal{N}_{\hat{\imath}, \hat{\lambda}}(\mathcal{E})<\left\|C_{\hat{\imath}} e(t)\right\|<\kappa_{2} \mathcal{N}_{\hat{\imath}, \hat{\lambda}}(\mathcal{E}) \\ 1 & \text { if }\left\|C_{\hat{\imath}} e(t)\right\|>\kappa_{2} \mathcal{N}_{\hat{\imath}, \hat{\lambda}}(\mathcal{E})\end{cases}
$$

The constants $\kappa_{1}$ and $\kappa_{2}$ are to be chosen in relation with inequality (30), for instance, we can take

$$
\kappa_{1}=\frac{\sqrt{q}}{2 \beta_{3} \eta^{\prime}}, \quad \kappa_{2}=\frac{\sqrt{q}}{\beta_{3} \eta^{\prime}} .
$$

Notice first that we have $\mathcal{E}(t)=G(t) E(t)(E(t)$ being the solution of $(22 \mathrm{~b})$ ) and so $e(t)=G(t)^{-1} \mathcal{E}(t) e(0)$. Notice also that $G$ is the solution to a linear differential equation, so $G$ is defined for every time $t \geq 0$; next, some elementary calculation shows the following explicit formula for $G(t)$ :

$$
G(t)=1+e^{a(t)} \int_{0}^{t} b(s) e^{-a(s)} \mathrm{d} s
$$

where

$$
a(t)=\int_{0}^{t}\left(s_{2} \mu(s)-s_{1}(1-\mu(s))\right) \mathrm{d} s, \quad b(t)=s_{2} \mu(t) .
$$

From this formula and as $\mu(t) \in[0,1]$ for every $t \geq 0$, we can see that $G(t) \geq 1$ for every $t \geq 0$. Moreover, some elementary manipulation shows that if $\mu(t) \equiv 0$ for $t \in\left[t_{0}, T\right)$, we have $G(t)=e^{-s_{1}\left(t-t_{0}\right)}\left(G\left(t_{0}\right)-1\right)+1$; on the other hand, if $\mu \equiv 1$ on $\left[t_{0}, T\right)$, then $G(t)=$ $e^{s_{2}\left(t-t_{0}\right)} G\left(t_{0}\right)$. So if $T=+\infty$ and $\mu \equiv 0($ resp. $\mu \equiv 1)$ on $\left[t_{0},+\infty\right)$, the gain $G$ tends to 1 (resp. tends to $+\infty$ ) as $t$ tends to infinity.

We shall use the same Lyapunov function as in the previous section but it depends now on $\hat{x}$ and $\mathcal{E}$ :

$$
W_{m}(\hat{x}, \mathcal{E})=\inf _{\lambda \in \Lambda^{+}}\left(\hat{x}^{\mathrm{T}} P_{\lambda} \hat{x}+q \operatorname{Tr}\left(\mathcal{E}^{\mathrm{T}} \Pi_{\lambda}^{-1} \mathcal{E}\right)\right) \text {. }
$$

When differentiating $W_{m}$ along the trajectories of the system constituted by the equations (22a) and (31), we have to take into account the term which contains $\dot{G} / G$ in (31), so inequality (24) is changed into

$$
\begin{aligned}
& \dot{W}_{m}(\hat{x}(t), \mathcal{E}(t)) \leq \\
&-\left(\hat{x}^{\mathrm{T}} Q_{\hat{\imath}}^{\mathrm{T}} \hat{x}+q\left(\operatorname{Tr}\left(\mathcal{E}^{\mathrm{T}} \Pi_{\hat{\lambda}}^{-1} S_{\hat{\imath}} \Pi_{\hat{\lambda}}^{-1} \mathcal{E}\right)+2 \frac{\dot{G}}{G} \operatorname{Tr}\left(\mathcal{E}^{\mathrm{T}} \Pi_{\hat{\lambda}}^{-1} \mathcal{E}\right)\right)\right. \\
&\left.\quad-2 e^{\mathrm{T}} C_{\hat{\imath}}^{\mathrm{T}} L_{\hat{\imath}, \hat{\lambda}}^{\mathrm{T}} P_{\hat{\lambda}} \hat{x}\right) .
\end{aligned}
$$

In this inequality, denote by $\mathcal{N}_{\lambda}^{\prime}$ the matricial norm defined as $\mathcal{N}_{\lambda}^{\prime}(M)=\left(\operatorname{Tr}\left(M^{\mathrm{T}} \Pi_{\lambda}^{-1} M\right)\right)^{1 / 2}$. Let $l_{i, \lambda}>0$ be such that $\mathcal{N}_{i, \lambda}(M) \geq l_{i, \lambda} \mathcal{N}_{\lambda}^{\prime}(M)$ for every matrix $M$, then in the expression between parenthesis in the righthand member of inequality (33), we have

$$
\mathcal{N}_{\hat{\imath}, \hat{\lambda}}(\mathcal{E})+2 \frac{\dot{G}}{G} \mathcal{N}_{\hat{\lambda}}^{\prime}(\mathcal{E}) \geq \frac{1}{2} \mathcal{N}_{\hat{\imath}, \hat{\lambda}}(\mathcal{E})
$$

provided that

$$
\frac{\dot{G}}{G} \geq-\frac{1}{4} l_{\hat{\imath}, \hat{\lambda}} .
$$

Now, as $\mu(t), G(t) \geq 0$, from (32), we have

$$
\frac{\dot{G}}{G} \geq-s_{1} \frac{G-1}{G} \geq-s_{1}
$$

and so inequality (34) is true provided that $s_{1}$ is chosen as

$$
s_{1} \leq \frac{l_{i, \lambda}}{4} \quad \text { for every }(i, \lambda) \in \mathfrak{S} \times \Lambda^{+} ;
$$

as the set $\in \mathfrak{S} \times \Lambda^{+}$is compact, the infimum of the $l_{i, \lambda}$ 's is positive and one can find $s_{1}>0$ such that the above inequality is satisfied. Parameter $s_{1}$ being so chosen, the treatment of inequality (33) is then almost the same that the one of inequality (23). The only change comes from the fact that $e(t)$ is now equal to $G(t)^{-1} \mathcal{E}(t) e(0)$, but as $G(t) \geq 1$ for every $t \geq 0$, we have $\|e(t)\| \leq$ $\|\mathcal{E}(t)\|\|e(0)\|$; using the same arguments as in the proof of Theorem 3, we can prove that the derivative of $W_{m}$ is negative definite provided that $e(0)$ is chosen less than $\frac{\sqrt{q}}{\beta_{2}}$. So, we proved the following theorem:

Theorem 4 We make the same assumptions as in The- 
orem 3. For every $(\hat{x}, \mathcal{E}) \in \mathbf{R}^{n} \times \mathbf{R}^{n \times n}$, we choose

$$
\begin{gathered}
(i(\hat{x}, \mathcal{E}), \lambda(\hat{x}, \mathcal{E})) \in \underset{(i, \lambda) \in \mathfrak{S} \times \ell(\hat{x}, \mathcal{E})}{\arg \min }\left(2 \hat{x}^{\mathrm{T}} A_{K_{i, \lambda}}^{\mathrm{T}} P_{\lambda} \hat{x}\right. \\
\left.+\hat{x}^{\mathrm{T}} M_{i, \lambda} \hat{x}+q \operatorname{Tr}\left(2 \mathcal{E}^{\mathrm{T}} A_{L_{i, \lambda}}^{\mathrm{T}} \Pi_{\lambda}^{-1} \mathcal{E}+\mathcal{E}^{\mathrm{T}} \Pi_{\lambda}^{-1} N_{i, \lambda} \Pi_{\lambda}^{-1} \mathcal{E}\right)\right) .
\end{gathered}
$$

Then the feedback related to $(i(\hat{x}, \mathcal{E}), \lambda(\hat{x}, \mathcal{E}))$ stabilizes the system made up of (22a) and (31) locally exponentially provided that the norm of $e(0)$ is less than some constant $\eta(q / 2)$; this constant tends to infinity as $q$ tends to infinity.

Remark 6 For the tuning of the gain $G(t)$, we could use as in [4] the innovation which is defined as follows

$$
\mathcal{I}_{d}(t)=\int_{t-d}^{t}\|y(\tau)-\hat{y}(\tau)\|^{2} \mathrm{~d} \tau
$$

where $\hat{y}$ denotes the output of the observer: $\hat{y}(\tau)=$ $C_{\sigma(\tau)} \hat{x}(\tau)$. Then, the definition of $\mu$ becomes

$$
\mu(t)= \begin{cases}0 & \text { if } \mathcal{I}_{d}(t) \leq \kappa_{1} \mathcal{N}_{\hat{\imath}, \hat{\lambda}}(\mathcal{E}) \\ \in(0,1) & \text { if } \kappa_{1} \mathcal{N}_{\hat{\imath}, \hat{\lambda}}(\mathcal{E})<\mathcal{I}_{d}(t)<\kappa_{2} \mathcal{N}_{\hat{\imath}, \hat{\lambda}}(\mathcal{E}) \\ 1 & \text { if } \mathcal{I}_{d}(t)>\kappa_{2} \mathcal{N}_{\hat{\imath}, \hat{\lambda}}(\mathcal{E})\end{cases}
$$

\subsubsection{Analysis of the robustness}

We shall show now that the new system is robust with respect to some perturbation. Assume that at time $t_{0}$, system (1a) is perturbed for a brief moment, this amounts to a re-initialization of $x(t)$ (and so of $e(t)$ at $t=t_{0}$ ), thus we have $e\left(t_{0}^{+}\right)=e\left(t_{0}\right)+p$ where $p$ demotes the perturbation. Take a time $t>0$, we have

$$
\begin{aligned}
e\left(t+t_{0}\right) & =E\left(t+t_{0}\right) E\left(t_{0}\right)^{-1}\left(e\left(t_{0}\right)+p\right) \\
& =E\left(t+t_{0}\right) e(0)+E\left(t+t_{0}\right) E\left(t_{0}\right)^{-1} p \\
& =\frac{1}{G\left(t+t_{0}\right)} \mathcal{E}\left(t+t_{0}\right)\left(e(0)+G\left(t_{0}\right) \mathcal{E}\left(t_{0}\right)^{-1} p\right) .
\end{aligned}
$$

In equation (32), observe that if $\mu\left(t+t_{0}\right)=1$ for every $t>0$, then, as noticed above, $G\left(t+t_{0}\right)$ tends to infinity. Assume now that we have $\left\|C_{\sigma\left(t+t_{0}\right)} e\left(t+t_{0}\right)\right\|>$ $\kappa_{2} \mathcal{N}_{i, \lambda}\left(\mathcal{E}\left(t+t_{0}\right)\right)$ for every $t>0$, then we deduce that

$$
\begin{aligned}
\kappa_{2} \mathcal{N}_{i, \lambda}\left(\mathcal{E}\left(t+t_{0}\right)\right)< & \left\|C_{\sigma(t)} e\left(t+t_{0}\right)\right\| \\
& =\frac{1}{G\left(t+t_{0}\right)} \| \mathcal{E}\left(t+t_{0}\right)(e(0) \\
& \left.+G\left(t_{0}\right) \mathcal{E}\left(t_{0}\right)^{-1} p\right) \| \\
\leq & \frac{1}{G\left(t+t_{0}\right)}\left\|\mathcal{E}\left(t+t_{0}\right)\right\| \\
\leq & \cdot\left\|e(0)+G\left(t_{0}\right) \mathcal{E}\left(t_{0}\right)^{-1} p\right\| \\
&
\end{aligned}
$$

this last inequality implies that

$$
\kappa_{2} \leq \frac{k_{i, \lambda}}{G\left(t+t_{0}\right)}\left\|e(0)+G\left(t_{0}\right) \mathcal{E}\left(t_{0}\right)^{-1} p\right\|
$$

which is incompatible with the fact that $G\left(t+t_{0}\right)$ tends to infinity as $t$ tends to infinity. So there must exist a time $t_{1}$ such that $\left\|C_{\sigma\left(t_{1}+t_{0}\right)} e\left(t_{1}+t_{0}\right)\right\|<\kappa_{2} \mathcal{N}_{i, \lambda}\left(\mathcal{E}\left(t_{1}+t_{0}\right)\right)$, and after this time, the derivative of $W_{m}$ becomes again negative and the conclusion of Theorem 4 is valid.

In conclusion, our feedback is robust with respect to perturbations which do not occur too often.

\section{Illustrative example}

Before presenting an example, it is important to mention that it is not necessary to ensure a stabilizing law to determine all the possible values of the set $\Lambda^{+}$. Only one value is sufficient to guarantee the stability. So, a reasonable finite number of values ensures performances. Practically, a finite number have been used using a discretization of the set $\Lambda$. Note also that for a set of respectively $10,10^{2}, 10^{3}, 10^{4}$ values taken in $\lambda^{+}$, the elapsed time to compute the output feedback in Matlab using a Mac Book Pro are $t_{\text {elapsed }}=1.710^{-4}, 2.10^{-4}, 2.510^{-4}$, $7.10^{-4} \mathrm{~s}$.

Consider now a two mode switched system defined by:

$$
\begin{aligned}
& \left(A_{1}, B_{1}, C_{1}\right)=\left(\begin{array}{cc}
1 & 0 \\
1 & -1
\end{array}\right),\left(\begin{array}{l}
0 \\
1
\end{array}\right),\left(\begin{array}{ll}
0 & 1
\end{array}\right) \\
& \left(A_{2}, B_{2}, C_{2}\right)=\left(\begin{array}{cc}
-2 & 1 \\
0 & 1
\end{array}\right),\left(\begin{array}{l}
1 \\
0
\end{array}\right),\left(\begin{array}{ll}
1 & 0
\end{array}\right)
\end{aligned}
$$

Clearly each subsystem is not stabilizable and not detectable. However, in a switched framework, choosing as 
design parameters $Q_{1}=Q_{2}=\mathrm{Id}, R_{1}=2, R_{2}=1$, $S_{1}=\left(\begin{array}{cc}10 & 0 \\ 0 & 1\end{array}\right), S_{2}=\left(\begin{array}{cc}1 & 0 \\ 0 & 10\end{array}\right), T_{1}=T_{2}=0.01$, the set $\Lambda^{+}$is non empty and the dynamic output feedback law can be applied.

In order to make some comparisons, Figure 1 shows the state space trajectories from different initial states taken on a ball of radius 5 and applying three types of controls:

- The open loop optimal solutions of the LQ problem (red) computed numerically [20];

- The dynamic state feedback law of the LQ problem (see Theorem 1) when full state measurement is available (green);

- The dynamic output feedback law (see Theorem 4) of the LQG problem when the state is partially measure through $y=C_{\sigma} x$.

The chosen parameters for the adaptive observer are:

$$
\begin{gathered}
q=3, \\
s_{1}=1, \quad s_{2}=100 \\
\mu(t)= \begin{cases}0 & \text { if } \mathcal{I}_{d}(t) \leq 0.1 \times \mathcal{N}_{\lambda}^{\prime}(\mathcal{E}) \\
\in(0,1) & \text { if } 0.1 \times \mathcal{N}_{\lambda}^{\prime}(\mathcal{E})<\mathcal{I}_{d}(t)<\mathcal{N}_{\lambda}^{\prime}(\mathcal{E}) \\
1 & \text { if } \mathcal{I}_{d}(t)>\mathcal{N}_{\lambda}^{\prime}(\mathcal{E}) .\end{cases}
\end{gathered}
$$

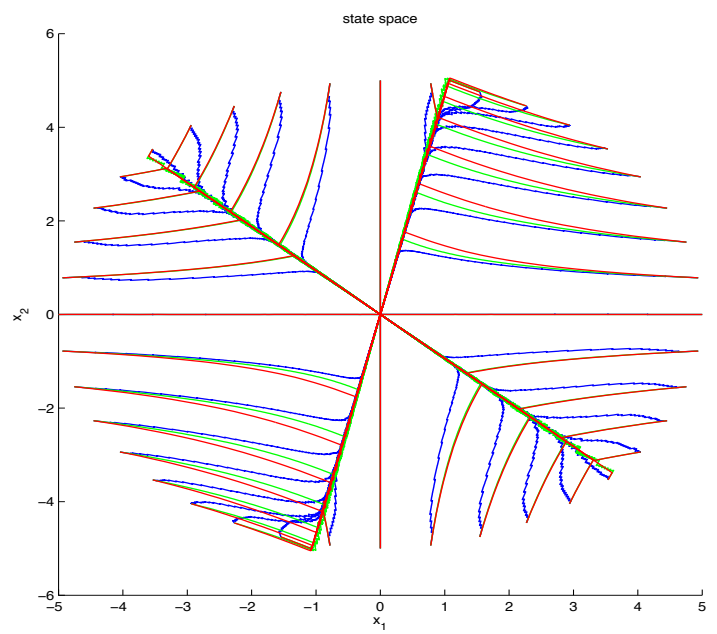

Figure 1. State space trajectories: (red) optimal solution; (green) dynamic state feedback ; (blue) dynamic output feedback $\left(\left\|e_{0}\right\|=0\right)$

We can see that the first two type of trajectories match well together (red and green lines). For the last one, we have deliberately set the initial estimated error to zero and $\mathcal{E}(0)=\mathrm{Id}$, then we can observe that the obtained trajectories are not so far from the optimal. Clearly a

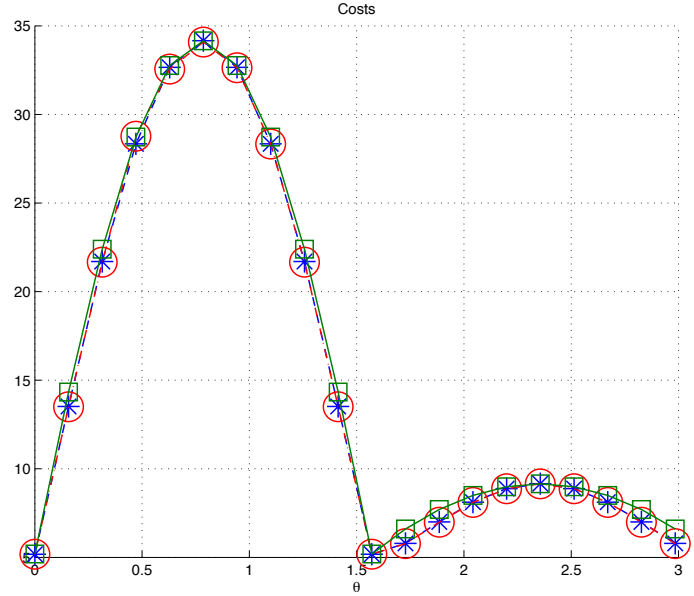

Figure 2. Cost comparison: (red circle) optimal, (blue diamond ) dynamic output feedback, (green star) dynamic output feedback, (green square) Lyapunov function $1 / 2 V_{m}$

tradeoff is made between estimation and control as it is implied by the design of Lyapunov function $W_{m}$.

Figure 2 shows the optimal cost, the costs associated respectively to the dynamic state and output feedback law, and the Lyapunov function $V_{m}$ of section 2 , the $x$ axis represents the polar angle $\theta$ of initial states. It can be observed that the costs associated to the state (output) feedback laws are very close to the cost of the optimal numerical solution. Of course, the essential difference is that the numerical solution is an open loop control. It is obvious in this case that the Lyapunov function $V_{m}$ is really a good approximation of the value function as invoked in section 2 .
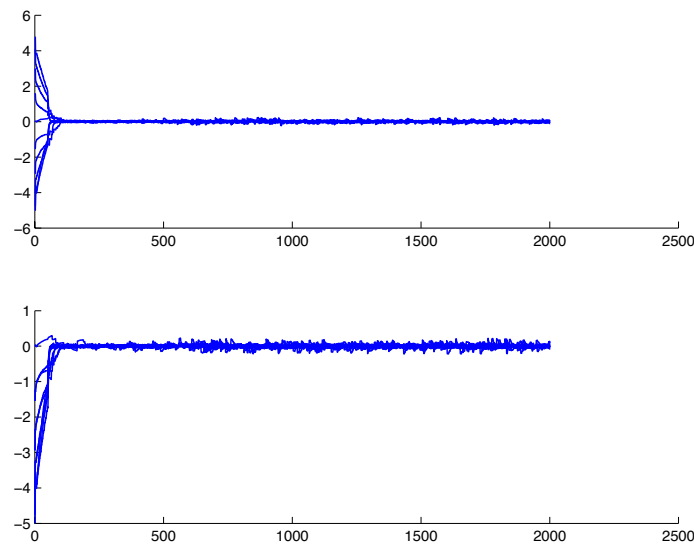

Figure 3. Estimation errors on the state $e=\hat{x}-x$ in presence of measurement noise.

Figure 4 shows now the dynamic output feedback law when the state is partially measure through $y=C_{\sigma} x+v$ 


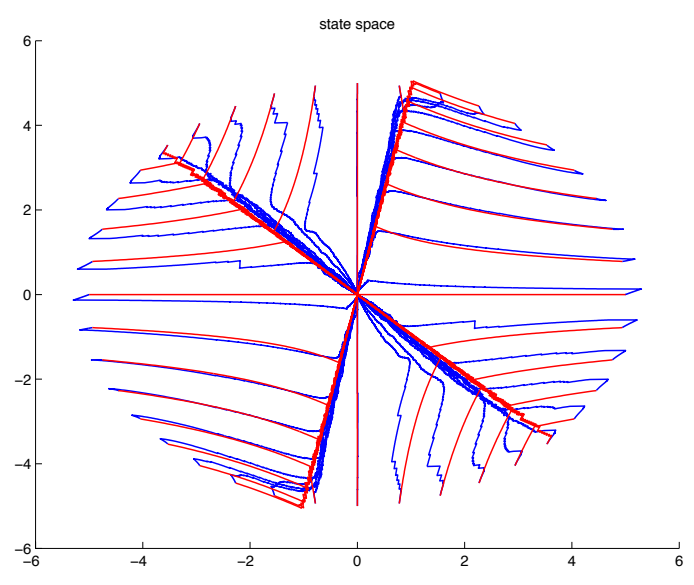

Figure 4. State space trajectories: (red) dynamic state feedback ; (blue) dynamic output feedback with measurement noises $\left(\left\|e_{0}\right\|=5\right)$

where noise $v$ is defined by an uniform law on $[-0.1,0.1]$. For this simulation, the chosen initial condition for $\hat{x}$ is the origin then the error norm $\|e(0)\|=5$. The convergence of the estimation error is depicted on Figure 3. As for a single linear system, the weight matrices can be used to modify the rate of convergence. On Figure 4, we can observe in comparison with the trajectories of the state feedback that the dynamic output feedback is robust with respect to the measurement noises. In view of this simulation, it seems that future works might concern a proof for stochastic stability.

\section{Application to power converters}

A privileged application of switched systems is certainly the DC-DC converters [7]. Their common aim is to convert a given input voltage to an another output voltage.

These class of systems can be described by switched affine systems of the form:

$$
\dot{x}=A_{i} x+B_{i} v
$$

where $v$ is a constant input and $i \in \mathfrak{S}$.

One way to address the control of these systems is to take the convex combination of its velocities (see for instance the remark concerning the density theorem in section 2). This model is often mentioned as the average model. Their equilibria are then directly related to the set: $X_{\text {ref }}=\left\{x: \sum_{i} \lambda_{i}\left(A_{i} x+B_{i} v\right)=0, \lambda \in \Lambda\right\}$.

We now show that the proposed design can be adapted to this class of systems. To illustrate the idea, we take for example a buck boost converter defined by two modes

$$
\begin{aligned}
& \left(A_{1}, B_{1}, C_{1}\right)=\left(\begin{array}{cc}
-R / L & 0 \\
0 & -1 / R_{0} C_{0}
\end{array}\right),\left(\begin{array}{c}
1 / L \\
0
\end{array}\right),\left(\begin{array}{ll}
1 & 0
\end{array}\right) \\
& \left(A_{2}, B_{2}, C_{2}\right)=\left(\begin{array}{cc}
-R / L & -1 / L \\
1 / C_{0} & -1 / R_{0} C_{0}
\end{array}\right),\left(\begin{array}{l}
0 \\
0
\end{array}\right),\left(\begin{array}{ll}
1 & 0
\end{array}\right)
\end{aligned}
$$

The state $\left(x_{1}, x_{2}\right)$ are respectively the current in the inductor and the output voltage. It is assumed that the current $x_{1}$ is measured and constitutes the output $y$. For a given equilibrium $x_{\text {ref }} \in X_{\text {ref }}$, consider the following augmented systems obtained by introducing an additional variable $x_{3}=\int\left(x_{1}-x_{1 r e f}\right) \mathrm{d} t$ and an additional control input:

$$
\begin{aligned}
\dot{x} & =A_{i} x+B_{i} v+B_{i} u_{i} \\
\dot{x}_{3} & =x_{1}-x_{1 \text { ref }}
\end{aligned}
$$

The rule of the integrator is to ensure a zero steady state error on the controlled variable $x_{1}$. This is directly inspired from the LQI design problem which extends the LQ design in the case where the control goal is not the origin. The observer is then

$$
\begin{aligned}
\dot{\hat{x}} & =A_{i} \hat{x}+B_{i} v+B_{i} u_{i}-L_{i}\left(C_{i} \hat{x}-y\right) \\
\dot{\hat{x}}_{3} & =\hat{x}_{1}-x_{1 \text { ref }}
\end{aligned}
$$

After the change of variable, $x_{e}=\left(x-x_{r e f}, x_{3}\right)$, the augmented system is rewritten in the new coordinates as well as the observer. The control Lyapunov function is now given by:

$$
W_{m}\left(\hat{x}_{e}, \mathcal{E}\right) \triangleq \inf _{\lambda \in \Lambda^{+}}\left({\hat{x_{e}}}^{\mathrm{T}} P_{\lambda} \hat{x}_{e}+q \operatorname{Tr}\left(\mathcal{E}^{\mathrm{T}} \Pi_{\lambda}^{-1} \mathcal{E}\right)\right)
$$

where $\hat{x}_{e}$ is the estimated value of $x_{e}$ and where $\mathcal{E}$ follows (31). Thus, the feedback law is now depending on $\left(\hat{x}_{e}, \mathcal{E}\right)$.

Remark 7 Note that the dynamics of $x_{e}$ is determined by $\dot{x}_{e}=\sum_{i} \lambda_{i}\left(A_{i} x_{e}+B_{i} u_{i}+d_{i}\right)$ where the terms $d_{i}=$ $\left(A_{i} x_{r e f}+B_{i} v\right)$. Obviously, the $d_{i}$ 's can be regarded as measured perturbations but a perturbation rejection analysis for the state dynamics in this context has not yet been performed and is out of the scope of the present paper. However, one can still guarantee the semi global stability property of the output feedback if the set $\Lambda^{+}$is restricted to the singleton $\left\{\lambda^{\text {ref }}\right\}$ where $\lambda^{\text {ref }}$ is the convex combination associated to $x_{\text {ref }}\left(i . e \sum_{i} \lambda_{i}^{r e f}\left(A_{i} x_{r e f}+B_{i} v\right)=0\right)$. This is obvious since in this case $d=\sum_{i} \lambda_{i}^{\text {ref }} d_{i}$ is zero and a decreasing descent direction for the control Lyapunov function $x_{e}^{T} P_{\lambda^{r e f}} x_{e}$ always exists as proved in Theorem 1. Of course, this restriction is detrimental for the performances but it makes the algorithm more simple to apply. In the sequel, we do not make this restriction. 


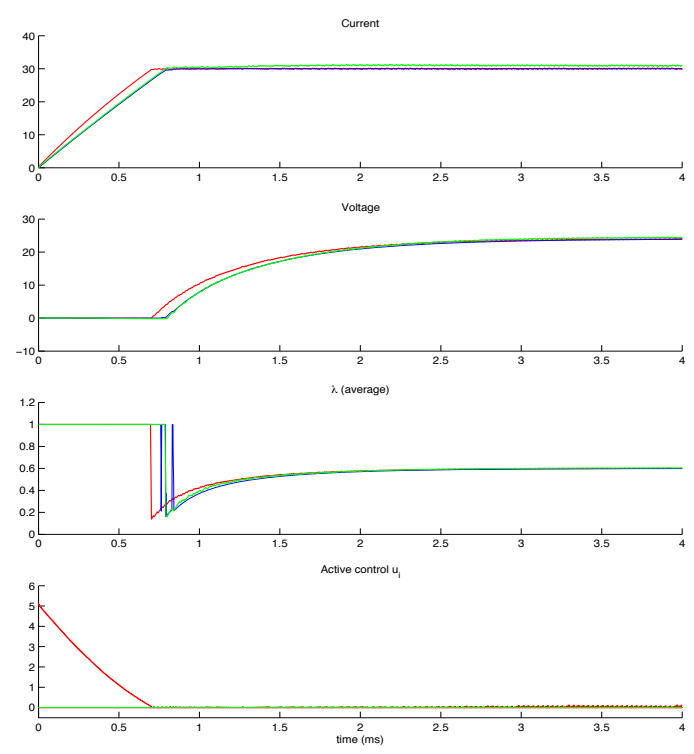

Figure 5. Startup of the Buck-Boost converter (blue: numerical optimal solution ; green: without active control $\left(R_{i}=10^{8}\right)$; red: with active control $\left.\left(R_{i}=10^{3}\right)\right)$ : time evolution of the voltage, the current, switching control $\lambda \in\left[\begin{array}{ll}0 & 1\end{array}\right]$ and of the active control $u_{i}$ in presence of measurement noise

Figure 5 shows the startup from zero initial conditions of the converter to reach the reference $x_{r e f}=(30,24)$. The control weight matrices are stated respectively to $R_{i}=10^{3}$ for the red line and to $R_{i}=10^{8}$ for the green line. In this latter case, the additional control input is almost identically zero and we retrieve the initial switched problem without additional input $u_{i}$. So, in order to make comparisons, we add, in blue, the numerical optimal solution corresponding to the minimization of $\int_{0}^{+\infty}\left(x-x_{r e f}\right) Q_{i}\left(x-x_{r e f}\right) d t$ subject to (36) with $u_{i}=0, \forall t, i=1,2$. It can be observed when no active control is used (i.e. $R_{i}=10^{8}$ ) that the result obtained is really close to the optimal solution (the curves overlap).

When an active control is considered $\left(R_{i}=10^{3}\right)$, a comparison with the other cases shows that it may be interesting to add this active control to improve performances. Finally, when sliding mode occurs (chattering), we have computed the equivalent switching control $\lambda_{1}$ as shown on Figure(5) (recall that $\lambda_{2}=1-\lambda_{1}$ ).

Figure 6 shows the convergence of the state error estimation from different initial positions taken in the ball $B\left(x_{r e f},\left\|x_{r e f}\right\|\right)$ with $\hat{x}(0)=x_{r e f}$, in presence of measurement noise defined by a uniform law on $[-1,1]$.

Simulation parameters: $v=20, R_{0}=2, R=R_{0} / 25$, $L=0.5 e^{-3}, C=0.5 e^{-3}$. Design parameters: $Q_{1}=$ $Q_{2}=\operatorname{diag}\left[\begin{array}{lll}200 & 1 & 0.01\end{array}\right], R_{1}=R_{2}=1000, S_{1}=S_{2}=$ $\operatorname{diag}\left[1\right.$ 100], $T_{1}=T_{2}=1, q=1, s_{1}=1, s_{2}=100$.
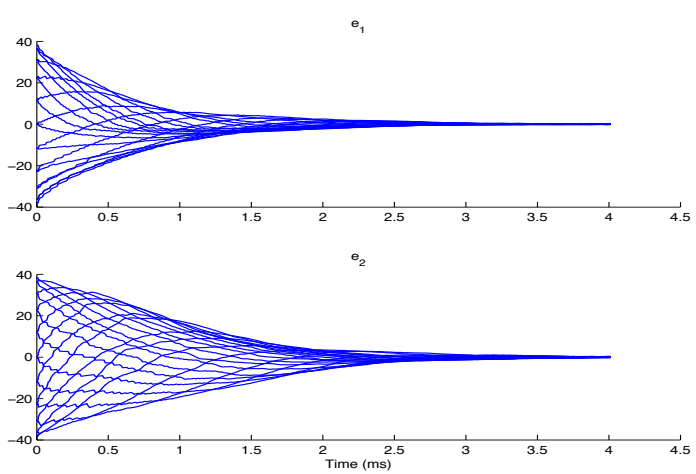

Figure 6. State error vs time in presence of measurement noise (uniform law on $[-1,1]$ )

Measurement noise : $y=C_{i} x+n_{u}$ where $n_{u}$ follows a uniform law on $[-1,1]$.

\section{Conclusion}

A dynamic output feedback has been proposed for switched linear systems that uses the LQG design procedure. The dynamic output feedback can be applied even if the subsystems are not stabilizable and not detectable. The only condition that is required, is the existence of at least one controllable and observable convex combination of the subsystems. It has been shown that the output regulator makes the stability of the system semi global with respect to the estimate error and allows perturbation rejection. Moreover, once the estimation error has converged, performances are guaranteed by the sub optimality of the dynamic state feedback presented in [21]. A proof concerning stochastic stability remains to produce in presence of noises. Finally, it has been shown that the design procedure can be applied to the large class of power converters.

\section{References}

[1] H. Axelsson, Y. Wardi, M. Egerstedt, and E.I. Verriest. Gradient descent approach to optimal mode scheduling in hybrid dynamical systems. Journal of Optimization Theory and Applications, 136(2):167-186, 2008.

[2] A. Bacciotti and L. Rosier. Liapunov Functions and Stability in Control Theory. Communications and Control Engineering. Springer, 2006.

[3] S.C. Bengea and R. A. DeCarlo. Optimal control of switching systems. Automatica, 41(1):11-27, 2005.

[4] N. Boizot, E. Busvelle, and J.-P. Gauthier. An adaptive highgain observer for nonlinear systems. Automatica, 46(9):14831488, 2010.

[5] P. Colaneri, J.C. Geromel, and A. Astolfi. Stabilization of continuous-time switched nonlinear systems. Systems \& Control Letters, 57(1):95-103, 2008.

[6] J. Daafouz, P. Riedinger, and C. Iung. Stability analysis and control synthesis for switched systems: a switched Lyapunov function approach. IEEE Transactions on Automatic Control, 47(11):1883-1887, Nov 2002. 
[7] G. S. Deaecto and et al. Switched affine systems control design with application to dc-dc converters. IET Control Theory and Applications, 4(7):1201-1210, 2010.

[8] G.S. Deaecto and J. Geromel, J.C.and Daafouz. Dynamic output feedback $\mathcal{H}_{\infty}$ control of switched linear systems. Automatica, 47(8):1713-1720, 2011.

[9] G.S. Deaecto, J.C. Geromel, and J. Daafouz. Switched state-feedback control for continuous time-varying polytopic systems. International Journal of Control, 84(9):1500-1508, 2011.

[10] N. Furukawa. Optimality conditions in nondifferentiable programming and their applications to best approximations. Applied Mathematics and Optimization, 9(1):337-371, 1982.

[11] J.C. Geromel and P. Colaneri. Stability and stabilization of discrete time switched systems. International Journal of Control, 79(7):719-728, 2006.

[12] J.C. Geromel, P. Colaneri, and P. Bolzern. Dynamic output feedback control of switched linear systems. IEEE Transactions on Automatic Control, 53(3):720-733, April 2008.

[13] J.C. Geromel, G.S. Deaecto, and J. Daafouz. Suboptimal switching control consistency analysis for switched linear systems. IEEE Transactions on Automatic Control, 58(7):1857-1861, July 2013.

[14] B. Ingalls, E.D. Sontag, and Y. Wang. An infinite-time relaxation theorem for differential inclusions. Proceedings of the American Mathematical Society, 131(2):487-499, 2003.

[15] V. Kučera. A review of the matrix Riccati equation. Kybernetika, 9(1):42-61, 1973.

[16] P. Lancaster and L. Rodman. Algebraic Riccati Equations. Oxford science publications. Clarendon Press, 1995.

[17] H. Lin and P.J. Antsaklis. Stability and stabilizability of switched linear systems: A survey of recent results. IEEE Transactions on Automatic Control, 54(2):308-322, Feb 2009.

[18] B. Lincoln and A. Rantzer. Relaxing dynamic programming. IEEE Transactions on Automatic Control, 51(8):1249-1260, 2006.

[19] D. Patino, P. Riedinger, and C. Iung. Practical optimal state feedback control law for continuous-time switched affine systems with cyclic steady state. International Journal of Control, 82(7):1357-1376, 2009.

[20] P. Riedinger and I.-C. Morărescu. A numerical framework for optimal control of switched input affine nonlinear systems subject to path constraint. Mathematics and Computers in Simulation, 95(0):63 - 77, 2014.

[21] P. Riedinger and J.-C. Vivalda. An LQ sub-optimal stabilizing feedback law for switched linear systems. In Proceedings of the 17th International Conference on Hybrid Systems: Computation and Control, 2014.

[22] C. Seatzu, D. Corona, A. Giua, and A. Bemporad. Optimal control of continuous-time switched affine systems. IEEE Transactions on Automatic Control, 51(5):726-741, 2006.

[23] M. Shahid Shaikh and P.E. Caines. On the hybrid optimal control problem: Theory and algorithms. IEEE Transactions on Automatic Control, 52(9):1587-1603, 2007.

[24] X. Xu and P.J. Antsaklis. Optimal control of switched systems based on parameterization of the switching instants. IEEE Transactions on Automatic Control, 49(1):2-16, 2004.

[25] W. Zhang, J. Hu, and A. Abate. On the value functions of the discrete-time switched LQR problem. IEEE Transactions on Automatic Control, 54(11):2669-2674, 2009.
[26] X. Zhao, S. Yin, H. Li, and B. Niu. Switching stabilization for a class of slowly switched systems. IEEE Transactions on Automatic Control, To appear. 